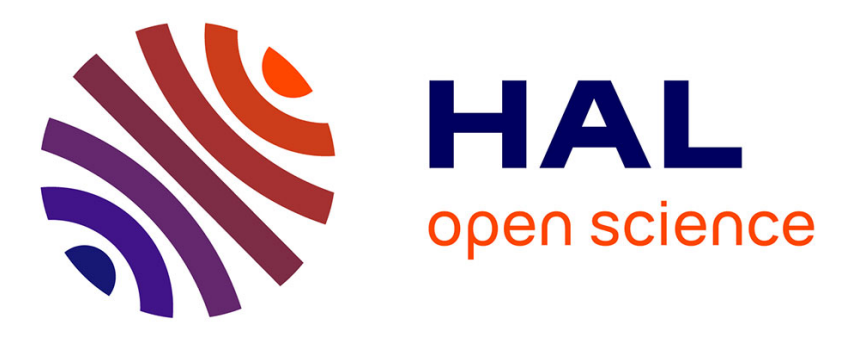

\title{
Influence of subthalamic deep-brain stimulation on cognitive action control in incentive context
}

Jean-François Houvenaghel, Joan Duprez, Soizic Argaud, Florian Naudet, Thibaut Dondaine, Gabriel Hadrien Robert, Sophie Drapier, Claire Haegelen, Pierre Jannin, Dominique Drapier, et al.

\section{To cite this version:}

Jean-François Houvenaghel, Joan Duprez, Soizic Argaud, Florian Naudet, Thibaut Dondaine, et al.. Influence of subthalamic deep-brain stimulation on cognitive action control in incentive context. Neuropsychologia, 2016, 91, pp.519-530. 10.1016/j.neuropsychologia.2016.09.015 . hal-01378672

\section{HAL Id: hal-01378672 \\ https://hal.science/hal-01378672}

Submitted on 14 Nov 2016

HAL is a multi-disciplinary open access archive for the deposit and dissemination of scientific research documents, whether they are published or not. The documents may come from teaching and research institutions in France or abroad, or from public or private research centers.
L'archive ouverte pluridisciplinaire HAL, est destinée au dépôt et à la diffusion de documents scientifiques de niveau recherche, publiés ou non, émanant des établissements d'enseignement et de recherche français ou étrangers, des laboratoires publics ou privés. 


\section{Influence of subthalamic deep-brain stimulation on cognitive action control in incentive context}

Jean-François Houvenaghel ${ }^{\mathrm{a}, \mathrm{b}}$, Joan Duprez ${ }^{\mathrm{a}}$, Soizic Argaud $^{\mathrm{a}, \mathrm{c}}$, Florian Naudet ${ }^{\mathrm{a}, \mathrm{d}, \mathrm{e}}$, Thibaut Dondaine $^{\mathrm{a}}$, Gabriel Hadrien Robert ${ }^{\mathrm{a}, \mathrm{e}}$, Sophie Drapier ${ }^{\mathrm{a}, \mathrm{b}}$, Claire Haegelen ${ }^{\mathrm{f}, \mathrm{g}}$, Pierre Jannin ${ }^{\mathrm{g}}$, Dominique Drapier ${ }^{\mathrm{a}, \mathrm{e}}$, Marc Vérin ${ }^{\mathrm{a}, \mathrm{b}}$, Paul Sauleau ${ }^{\mathrm{a}, \mathrm{h}}$

a "Behavior and Basal Ganglia" research unit (EA 4712), University of Rennes 1, Rennes, France

${ }^{\mathrm{b}}$ Department of Neurology, Rennes University Hospital, Rennes, France

c “Neuroscience of Emotion and Affective Dynamics" laboratory, Department of Psychology and Educational Sciences / Swiss Center for Affective Sciences, Campus Biotech, University of Geneva, Geneva, Switzerland

${ }^{\mathrm{d}}$ Clinical Investigation Center (INSERM 0203), Department of Pharmacology, Rennes

University Hospital, Rennes, France

${ }^{\mathrm{e}}$ Department of Psychiatry, Rennes University Hospital, Rennes, France

${ }^{\mathrm{f}}$ Department of Neurosurgery, Rennes University Hospital, Rennes, France

g “MediCIS” laboratory (UMR 1099 LTSI), INSERM/University of Rennes, Rennes, France

${ }^{\mathrm{h}}$ Department of Neurophysiology, Rennes University Hospital, Rennes, France

Corresponding author: Jean-François Houvenaghel, Service de Neurologie, CHU

Pontchaillou, 2 rue Henri Le Guilloux, 35033 Rennes Cedex, France. Phone: +(33)2 992898

42, Fax: +(33)2 992841 32, E-mail: jeanfrancois.houvenaghel@chu-rennes.fr 


\title{
ACCEPTED MANUSCRIPT
}

Word count: Abstract: 232 - Main text: 7144 . 30 references, 3 tables, 12 figures; no

supplemental data

\begin{abstract}
Subthalamic nucleus deep-brain stimulation (STN-DBS) is an effective treatment in Parkinson's disease (PD), but can have cognitive side effects, such as increasing the difficulty of producing appropriate responses when a habitual but inappropriate responses represent strong alternatives. STN-DBS also appears to modulate representations of incentives such as monetary rewards. Furthermore, conflict resolution can be modulated by incentive context. We therefore used a rewarded Simon Task to assess the influence of promised rewards on cognitive action control in 50 patients with PD, half of whom were being treated with STN-
\end{abstract} DBS. Results were analyzed according to the activation-suppression model. We showed that STN-DBS (i) favored the expression of motor impulsivity, as measured with the Barratt Impulsiveness Scale, (ii) facilitated the expression of incentive actions as observed with a greater increase in speed according to promised reward in patients with versus without DBS and (iii) may increase impulsive action selection in an incentive context. In addition, analysis of subgroups of implanted patients suggested that those who exhibited the most impulsive action selection had the least severe disease. This may indicate that patients with less marked disease are more at risk of developing impulsivity postoperatively. Finally, in these patients, incentive context increased the difficulty of resolving conflict situations. As a whole, the current study revealed that in patients with PD, STN-DBS affects the cognitive processes involved in conflict resolution, reward processing and the influence of promised rewards on conflict resolution.

Keywords: reward, cognitive control, Simon Task, Parkinson's disease, subthalamic nucleus, deep brain stimulation 


\section{ACCEPTED MANUSCRIPT}

\section{INTRODUCTION}

Subthalamic nucleus deep brain stimulation (STN-DBS) is an effective treatment for Parkinson's disease (PD), bringing about a well-documented improvement in motor symptoms. However, it has become clear that it also induces cognitive changes, including modifications in both cognitive action control and reward processing (Jahanshahi et al., 2015; Palminteri et al., 2013; Zavala et al., 2015).

Cognitive action control refers to a set of cognitive processes that favor the production of goal-directed actions according to either internal objectives or environmental requirements. It is classically assessed using conflict tasks such as the Simon Task (Simon, 1990) in which participants have to respond according to the relevant-color information while ignoring irrelevant-location information of the stimulus. According to the dual-route model, the stimuli in these tasks have both relevant and irrelevant dimensions, which activate in parallel both appropriate and inappropriate actions (Kornblum et al., 1990). When the relevant and irrelevant dimensions activate the same motor program (congruent situation), the action is facilitated. By contrast, when the relevant and irrelevant dimensions activate different motor programs (noncongruent situation), cognitive effort is required to resolve the conflict by suppressing the inappropriate activation in order to favor the appropriate response. This cognitive effort is represented by the congruence effect, which is reflected by increases in reaction time and error rate in noncongruent versus congruent situations. The activation-suppression model gives insights into the cognitive processes involved in cognitive action control (Ridderinkhof, 2002a, 2002b, 2004). This model postulates that the cognitive action control used to resolve conflict situations is temporally dynamic. More specifically, the inhibitory control that can selectively suppress inappropriate activations takes time to become active and effective. Fast responses are therefore less controlled than slow ones. Analysis of 


\section{ACCEPTED MANUSCRIPT}

the distribution of performances across response times allows us to examine impulsive response selection and suppression. The conditional accuracy function (CAF), which represents the accuracy rate as a function of response speed, assesses impulsive action selection, which is portrayed by the amount of fast errors in noncongruent situation. Delta plots, which represent the congruence effect as a function of response speed, investigate impulse response suppression efficiency, which is estimated by the slope of the congruence effect for the slowest responses.

Although there is growing evidence in the literature for the STN's involvement in cognitive action control (Jahanshahi et al., 2015; Zavala et al., 2015), its precise role in conflict situations is still subject to debate. Using the activation-suppression model, Wylie, Ridderinkhof, Elias et al. (2010) demonstrated that STN-DBS induces impulsive action selection, but also improves impulse suppression. Stimulated patients with PD produced more fast, erroneous responses in a conflict situation, but when they took more time before answering, it was easier for them to suppress the impulsive response. However, to our knowledge these results have not been replicated.

Reward processing refers to the cognitive system that generates incentive motivation mediated by an appetitive stimulus. STN-DBS has recently been demonstrated to modulate reward processing (Palminteri et al., 2013; Wagenbreth et al., 2015). Compared with an inactive stimulation condition, DBS activation led patients to produce more effort (squeezing a handgrip) to achieve high versus low promised rewards (Palminteri et al., 2013). This suggests that STN-DBS can boost the incentive effect of promised rewards.

In addition, incentive motivation induced by anticipation of the reward modulates conflict resolution (Aarts et al., 2014; Di Rosa et al., 2015; Padmala and Pessoa, 2011). However, 


\section{ACCEPTED MANUSCRIPT}

predictions regarding the beneficial or detrimental effect of promised rewards on cognitive action control are hard to establish and probably task specific. Some authors have shown that promised rewards impair cognitive action control (Padmala and Pessoa, 2010), but others have observed a facilitatory effect (Padmala and Pessoa, 2011), while several authors relying on behavioral data have found no significant effect whatsoever (Aarts et al., 2014; van den Berg et al., 2014). Accordingly, an increase in incentive motivation induced by STN-DBS could have either a detrimental or a beneficial effect on cognitive action control. Predictions about how the interaction between cognitive control and incentive motivation might be modulated by STN-DBS therefore seem highly speculative at present.

The basal ganglia seem to be closely involved in these processes, as confirmed by both imaging (Aarts et al., 2014; Padmala and Pessoa, 2011) and behavioral studies in patients with PD which showed no modulation of performance according to the incentive context (Di Rosa et al., 2015). However, the specific role of the STN in the interaction between incentive motivation and conflict resolution has not yet been investigated. Given that STN-DBS influences both cognitive action control and the incentive effect of promised rewards, we might expect it to modulate the influence of incentives on conflict resolution processes. Experimental studies featuring an On-Off design are particularly useful for assessing the effects of electrical stimulation of the STN. However, they yield little information about the global treatment including the specific effect of electrical stimulation and the potential lesioning effect of electrode implantation on cognitive processing. To assess the impact of STN-DBS on the interaction between cognitive action control and incentive motivation, we therefore administered a rewarded Simon Task to 25 patients with PD who were not undergoing STN-DBS and 25 patients who were receiving STN-DBS, and compared the performances of these two groups in the light of the dual-route model (Kornblum et al., 1990) and the activation-suppression model (Ridderinkhof, 2002a, 2002b, 2004). 


\section{ACCEPTED MANUSCRIPT}

\section{METHODS}

\subsection{Participants}

Two groups of 25 patients with idiopathic PD (Hughes et al., 1992) took part in the study. The first group (10 men, 15 women) was composed of patients who were candidates for STN-DBS (without STN-DBS group). The second group (12 men, 13 women) was composed of patients who had already undergone surgery for bilateral STN-DBS at Rennes University Hospital, France (with STN-DBS group). These patients were assessed on average $30 \pm 24$ months after surgery. Standard selection criteria for surgery were applied to all patients (Welter et al., 2002).

All participants underwent a neuropsychological and psychiatric assessment. Dementia was excluded using the Mattis Dementia Rating Scale (MDRS; Mattis, 1988). Psychiatric disorders and severe depression were excluded using the French version of the MiniInternational Neuropsychiatric Interview (MINI 500; Sheehan et al., 1998) and the Montgomery-Åsberg Depression Rating Scale (MADRS; Montgomery \& Åsberg, 1979). Impulsivity was assessed on the Barratt Impulsiveness Scale (BIS-10; Baylé et al., 2000), apathy on the Apathy Evaluation Scale (AES; Marin, Biedrzycki, \& Firinciogullari, 1991) and anxiety on the State-Trait Anxiety Inventory (STAI; Spielberger, Gorsuch, \& Lushene, 1970). To assess executive functions, we used a neuropsychological battery that included a 2-minute version of the semantic (animals) and phonemic (letter p) verbal fluency test (Cardebat et al., 1990), Nelson's simplified version of the Wisconsin Card Sorting Test (MCST; Nelson, 1976), the Trail Making Test (TMT; Reitan, 1958) and the Stroop Test (Golden, 1978). PD severity was rated on the Unified Parkinson's Disease Rating Scale (UPDRS Part III), and the Hoehn and Yahr and Schwab and England scales. Clinical data are shown in Table 1. Both groups were comparable for sociodemographic characteristics, cognitive functioning (overall cognition and executive functions), behavioral/psychiatric characteristics (trait 


\section{ACCEPTED MANUSCRIPT}

impulsivity, anxiety, depression and apathy), disease duration and disease severity, as measured with the UPDRS-III, Hoehn and Yahr, and Schwab and England scales on medication. Groups differed solely on the Stroop Word score, levodopa equivalent daily dose (LEDD) and Schwab and England score off medication. The significant difference between the groups on the Stroop Word score cannot be interpreted as consequence of a general cognitive slowdown, as the Stroop Color score, the time taken to complete the TMT Part A or $\mathrm{B}$, and the overall reaction time in our incentive Simon Task did not differ between groups. In the without STN-DBS group, medication consisted of levodopa and dopaminergic agonists in 17 patients, and levodopa alone in eight patients. In the STN-DBS group, 19 patients were on levodopa and dopaminergic agonists, four on levodopa only and two on dopaminergic agonists only. All the patients remained under anti-parkinsonian medication when they performed the experimental task, to limit the effect of their motor symptoms. The experiment was approved by the local ethics committee of Rennes University Hospital and conducted in accordance with the Declaration of Helsinki and current French legislation (Huriet Act). 


\section{ACCEPTED MANUSCRIPT}

Table 1: Clinical data (mean $\pm S D$ ) and comparison between groups with and without STNDBS

$\mathrm{N}$

Without/With Without STN-

\begin{tabular}{lcccc} 
& STN-DBS & DBS & With STN-DBS & $p$ value \\
\hline$N$ Men/Women & & $10 / 15$ & $12 / 13$ & 0.57 \\
Age (years) & $25 / 25$ & $57.8 \pm 8.7$ & $57.8 \pm 6.9$ & 0.95 \\
\hline Education (years) & $25 / 25$ & $11.6 \pm 4.5$ & $10.6 \pm 3.7$ & 0.55 \\
MADRS & $25 / 25$ & $5.3 \pm 4.4$ & $4.6 \pm 4.6$ & 0.42
\end{tabular}

BIS-10

$21 / 22$

Total

$52.8 \pm 15.9 \quad 46.7 \pm 13.2 \quad 0.37$

Motor impulsivity

$15.8 \pm 8.3$

$13.4 \pm 6.6$

0.37

Cognitive impulsivity

$17.3 \pm 6.0$

$17.0 \pm 5.4$

0.85

Planning impulsivity

$19.6 \pm 5.5$

$16.3 \pm 6.3$

0.09

STAI State

$22 / 22$

$35.8 \pm 11.2$

$32.8 \pm 12.0$

0.29

STAI Trait

$22 / 23$

$41.4 \pm 10.0$

$39.9 \pm 11.1$

0.45

AES

$23 / 25$

$27.5 \pm 6.7$

$30.3 \pm 8.8$

0.53

MDRS

$25 / 25$

$139.3 \pm 3.7$

$139.8 \pm 4.3$

0.38

Stroop

Color

Word

Color-Word

$25 / 25$

$25 / 25$

$25 / 25$

Interference

$25 / 25$

TMT

Part A

25/25

$24 / 25$

$24 / 25$

B-A

MCST

Categories

Errors

Perseverations

Verbal fluency

\begin{tabular}{lcccc}
\multicolumn{1}{c}{ Semantic } & $25 / 25$ & $28.8 \pm 8.1$ & $27.7 \pm 7.8$ & 0.42 \\
\multicolumn{1}{c}{ Phonemic } & $25 / 25$ & $20.8 \pm 8.7$ & $20.6 \pm 6.3$ & 0.68 \\
\hline Disease duration (years) & $25 / 25$ & $11.6 \pm 3.6$ & $12.7 \pm 2.9$ & 0.15 \\
Age at disease onset (years) & $25 / 25$ & $46.5 \pm 7.7$ & $45.48 \pm 7.1$ & 0.69 \\
UPDRS-III on med - on stim & $-/ 25$ & - & $9.2 \pm 5.5$ & - \\
UPDRS-III on med - off stim & $25 / 25$ & $11.6 \pm 6.7$ & $16.16 \pm 8.9$ & 0.06 \\
UPDRS-III off med - on stim & $-/ 25$ & - & $21.6 \pm 11.4$ & - \\
UPDRS-III off med - off stim & $24 / 25$ & $34.4 \pm 15.3$ & $37.9 \pm 11.9$ & 0.37 \\
Schwab and England (\%) on med & $25 / 25$ & $91.6 \pm 9.2$ & $91.2 \pm 8.0$ & 0.68 \\
Schwab and England (\%) off med & $25 / 25$ & $68.8 \pm 13.0$ & $80.8 \pm 12.3$ & $<0.001$ \\
Hoehn and Yahr on med & $25 / 25$ & $1.2 \pm 1.1$ & $1.1 \pm 1.1$ & 0.54 \\
Hoehn and Yahr off med & $25 / 25$ & $2.4 \pm 0.9$ & $2.1 \pm 0.9$ & 0.33 \\
LEDD (mg/day) & $25 / 25$ & $1230.4 \pm 448.0$ & $858.1 \pm 456.2$ & $<0.0001$ \\
\hline
\end{tabular}

Note. MADRS = Montgomery and Asberg Depressive Rating Scale; BIS-10 = Barrat Impulsiveness Scale; STAI $=$ State-Trait Anxiety Inventory; AES = Apathy Evaluation Scale; MDRS = Mattis Dementia Rating Scale; TMT 


\section{ACCEPTED MANUSCRIPT}

$=$ Trail Making Test; MCST $=$ Modified Wisconsin Card Sorting Test; UPDRS $=$ Unified Parkinson's Disease Rating Scale; LEDD = levodopa equivalent daily dose. p.value were estimated by using Mann-Whitney U test

\subsection{Neurosurgery and Stimulation Settings}

Surgery was performed under local anesthesia, using MRI determination of the target, microrecording and intraoperative assessment of the clinical effects induced by stimulation. The correct position of the electrodes was checked postoperatively using a 3D CT brain scan. Quadripolar electrodes (3389; Medtronic, Minneapolis, MN, USA) were implanted bilaterally in all the patients. The mean coordinates of the selected contacts were $13.4 \pm 2.2 \mathrm{~mm}$ lateral, $3.3 \pm 2.4 \mathrm{~mm}$ below, and $2.3 \pm 1.9 \mathrm{~mm}$ posterior to the midpoint of the bicommissural (AC-PC) line. The mean parameters for the monopolar stimulation were $2.1( \pm 0.5) \mathrm{V}, 61.1( \pm$ 5.9) $\mu$ s and $130.7( \pm 2.9) \mathrm{Hz}$.

\subsection{Materials and Procedure}

Participants were first required to complete 32 trials of a classic Simon Task, to measure their mean reaction time (RT) at baseline. They then performed a rewarded Simon Task composed of 360 trails divided into five blocks of 72 trials.

All the stimuli were displayed on the black screen of a 23.6-inch digital monitor (Fig. 1). This screen was placed $90 \mathrm{~cm}$ away from the participant. Each trial began with a central cue displayed for $1000 \mathrm{~ms}$. This cue was a white cross in the baseline phase $\left(2.2 \times 2.2 \mathrm{~cm}, 1.02^{\circ}\right.$ visual angle), and a coin indicating the promised reward for each trial in the experimental phase. The coin was either $€ 1,1$ cent or a chimeric combination of the two (no reward; $€ 0$ ), as participants were explicitly told. Each coin had a diameter of $3.7 \mathrm{~cm}$ that subtended a visual angle of $2.35^{\circ}$. The cue was followed by a black screen for $700-1100 \mathrm{~ms}$, after which a blue or yellow circle (target) appeared on the right or left side of the screen. Each circle had a diameter of $5.5 \mathrm{~cm}$ that subtended a visual angle of $2.48^{\circ}$. The distance between the center of 


\section{ACCEPTED MANUSCRIPT}

the computer screen and the edge of the circle was a $6.50^{\circ}$ visual angle. Participants were asked to press a blue or yellow button on a keyboard, as quickly and accurately as possible, according to the color of the target and independently of its position. Thus, the position of the target defined the congruence of the trial (i.e., congruent: blue circle located on the side of the blue button; noncongruent: blue circle located on the side of the yellow button). The target disappeared as soon as participants pressed one of the two buttons. In the experimental phase, participants were instructed to press the button indicated by the color of the target as quickly and accurately as possible, in order to obtain the greatest reward, the size of the reward being proportionate to the response speed for the correct responses. In reality, the size of the reward for correct responses was calculated on a linear basis. To obtain the full promised reward, participants' RT had to be at least one sixth less than their baseline RT (e.g., if their baseline RT was $480 \mathrm{~ms}$, they only obtained the full reward if they responded correctly within $400 \mathrm{~ms}$ or less). A correct response with an RT equal to the baseline RT brought them $50 \%$ of the promised reward. Slower correct responses and incorrect responses were not rewarded. At the end of each trial, the amount of money they had won virtually since the beginning of the relevant experimental block was displayed for $1500 \mathrm{~ms}$, the maximum reward for each block being $€ 24.24$. The position of the color button was pseudorandomized across participants, and all experimental conditions were pseudorandomized, with an equal number of trials in each condition. At baseline, each participant performed 16 congruent trials and 16 noncongruent ones. In the experimental phase, each combination of congruence (congruent/noncongruent) and level of reward $(€ 1 / 1$ cent / $€ 0)$ was repeated 60 times per participant. 


\section{ACCEPTED MANUSCRIPT}

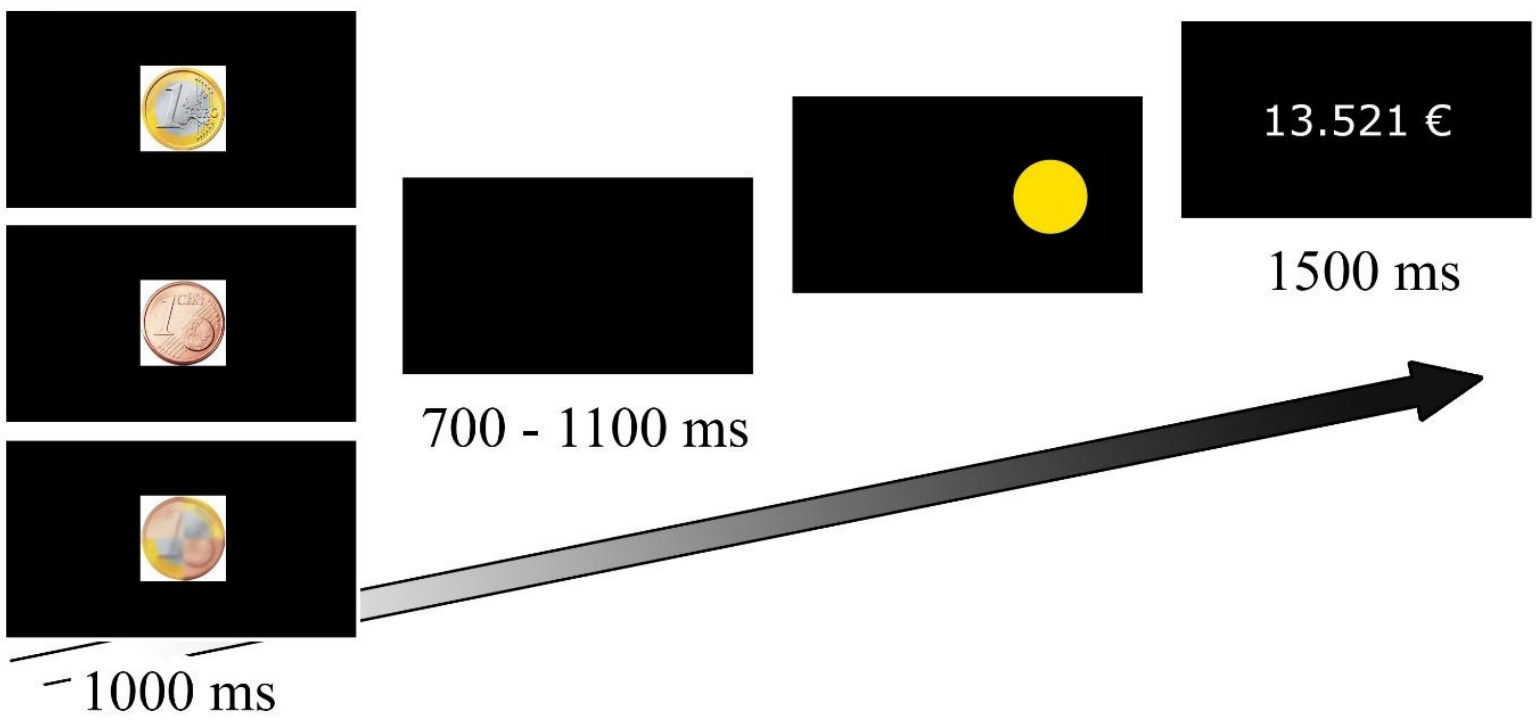

Figure 1: At the beginning of each trial, the incentive monetary cue ( $€ 0,1$ cent or $€ 1)$ was displayed for 1000 ms. After an interval of 700-1100 ms, a blue or yellow circle appeared on the left or right side of the screen, and remained there until participants pressed the blue or yellow button (as quickly and accurately as possible). At the end of each trial, the size of the reward accumulated since the start of the experimental block was displayed for $1500 \mathrm{~ms}$.

\subsection{Statistical Analyses}

Statistical analyses were performed using R (Version 3.1.0), implemented with the nlme (Pinheiro et al., 2015) and lme4 (Bates et al., 2015) packages. Only responses in the experimental phase with RTs between $200 \mathrm{~ms}$ and $1500 \mathrm{~ms}$ and within three standard deviations of the mean were analyzed. These cut-off points removed $1.3 \%$ of the whole dataset. As RTs were not normally distributed, they were first converted using logarithmic transformation. Statistical analyses were therefore based on response accuracy and the transformed RTs for correct responses. To avoid losing statistical power and to take interindividual variability into account, we used the whole dataset and not the averaged data per condition and per participant. A significance threshold of $p=0.05$ was used for all the statistical analyses. 


\section{ACCEPTED MANUSCRIPT}

We first analyzed the impact of STN-DBS on the influence of promised rewards on cognitive action control, using a linear mixed model for RTs and a nonlinear mixed model for response accuracy. In each model, the fixed effects were group (2 levels: without STN-DBS and with STN-DBS), congruence (2 levels: congruent and noncongruent) and reward (3 levels: $€ 1,1$ cent and $€ 0$ ), the covariate was LEDD and the random effect was participant. We then estimated the impact of STN-DBS on the influence of promised rewards on the temporal dynamics of cognitive action control. To assess impulsive action selection, we used CAF to plot response accuracy along RTs for each condition and participant, with each distribution divided into three quantiles (bins) according to the RTs. To analyze response accuracy, we used a nonlinear mixed model in which the fixed effects were group, congruence, reward and bin (3 levels: first, second and third bins), the covariate LEDD, and the random effect participant. In accordance with the activation-suppression model, we focused on the first bin to estimate impulsive action selection, as the first bin is thought to reflect this process better (Ridderinkhof, 2002a, 2002b, 2004). To assess selective response suppression, we focused on changes in the congruence effect for correct responses, according to the RTs for each reward and each participant, and again divided each distribution into three quantiles (bins). We then calculated the mean RT for each part of the distribution, resulting in individual Vincentized distributions. We then estimated the slope between each bin (delta plots, 2 slopes) and analyzed these slopes using a linear mixed model in which the fixed effects were group, reward and slope (2 levels: first slope and second slope), the covariate LEDD, and the random effect participant. Previous studies had suggested that the more negative the slope, the more effective the impulse suppression (Ridderinkhof, Forstmann, Wylie, Burle, \& van den Wildenberg, 2011). All p-values regarding the linear mixed models were computed using the anova function that uses F-tests while p-values for non-linear mixed models were computed using the Anova function that calculates Wald Chi-square tests (Anova $\{$ car $\}$ ). 


\section{ACCEPTED MANUSCRIPT}

\section{RESULTS}

This section is divided into two parts, each further divided into three subparts. The first part concerns the comparison of patients with and without STN-DBS, while the second part deals with the analysis of two subgroups of patients with STN-DBS. Each part begins with an analysis of the overall data, as classically reported when studying conflict tasks according to the dual-route model. The data are then analyzed according to the activation-suppression model in which we report the results of the CAF, followed by the delta plots. In each subpart we considered the effects of congruence and reward, and the interaction between the two, before proceeding to group comparisons. It should be noted that although LEDD differed between groups, it had no effect on either accuracy $\left(\chi^{2}=.02, p=.90\right)$ or $\mathrm{RT}\left(F_{(1,16633)}=.01, p\right.$ $=.90)$. We nevertheless introduced it as a covariate in each statistical model, to limit the potential influence of dopaminergic treatment. The tables showing the main statistical results are provided as Supplementary Data.

\subsection{Patients With Versus Without STN-DBS}

\subsubsection{Overall RT and accuracy}

When we considered all the participants, we observed the classic congruence effect (Congruence, $\mathrm{RT}, F_{(1,16633)}=1564.96, p<.0001 ;$ accuracy, $\left.\chi^{2}=352.25, p<.0001\right)$ confirming that the selection of correct responses was harder in the noncongruent condition than in the congruent one (Table 2). The promise of a reward reduced RT for correct responses $\left(\right.$ Reward, RT, $\left.F_{(2,16633)}=678.85, p<.0001\right)$, without affecting their accuracy (Reward, accuracy, $\chi^{2}=1.99, p=.37$ ). Responses were the fastest in the $€ 1$ condition (mean \pm $S D: 482 \pm 138 \mathrm{~ms})$ and responses in the 1 cent situation $(511 \pm 141 \mathrm{~ms})$ were faster than in the 


\section{ACCEPTED MANUSCRIPT}

nonrewarded situation (528 $\pm 142 \mathrm{~ms}$ ) (Reward, RT: $€ 0$ vs. 1 cent, $F_{(1,1114)}=93.21, p<$ $.0001 ; € 0$ vs. $€ 1, F_{(1,11122)}=660.81, p<.0001 ; 1$ cent vs. $\left.€ 1, F_{(1,11150)}=267.64, p<.0001\right)$. Furthermore, the promise of a reward modulated the congruence effect for both RT and accuracy (Congruence $\times$ Reward, RT, $F_{(2,16633)}=9.65, p=.008$; accuracy, $\chi^{2}=6.96, p=.03$ ). The congruence effect was greater in the $€ 1$ situation than in the 1 cent situation for both RT and accuracy (Congruence $\times$ Reward, RT, 1cent vs. $€ 1, F_{(1,11094)}=9.71, p=.002 ; € 0$ vs. 1 cent, $F_{(1,11058)}=1.09, p=.29 ; € 0$ vs. $\left.€ 1, F_{(1,11066)}=3.73, p=.05\right)($ Congruence $\times$ Reward, accuracy, 1 cent vs. $€ 1, \chi^{2}=6.69, p=.01 ; € 0$ vs. 1 cent, $\chi^{2}=1.36, p=.24 ; € 0$ vs. $€ 1, \chi^{2}=1.53$, $p=.21$; congruence effect on error rate: $€ 0=6.9 \%$; 1 cent $=6.2 \% ; € 1=8.5 \%$ ).

Group comparisons (Table 2) showed that the groups did not differ on overall RT (Group, $F_{(1 \text {, }}$ 47) $=0.01, p=.96)$ but did differ on overall accuracy (Group, $\left.\chi^{2}=8.15, p=.004\right)$. Patients with STN-DBS had a higher error rate (7.7\%) than patients without STN-DBS (4.2\%). The congruence effect did not differ between groups (Group $\times$ Congruence, RT, $F_{(1,16633)}=0.51, p$ $=.48$; accuracy, $\chi^{2}=1.25, p=.26$ ). The reduction in $\mathrm{RT}$ as the size of the reward increased was greater in patients with STN-DBS (Group $\times$ Reward, RT, $F_{(2,16633)}=35.93, p<.0001$; accuracy, $\chi^{2}=1.98, p=.37$ ) (Fig. 2). Despite faster action selection according to the promised reward by patients with STN-DBS, the cognitive effort required to control these fast responses did not differ between groups (Group $\times$ Congruence $\times$ Reward, $\mathrm{RT}, F_{(2,16633)}=$ $0.11, p=.94)$ 


\section{ACCEPTED MANUSCRIPT}

Table 2: Means and standard errors of the mean (SEMs) for reaction times and error rates for patients with and without DBS

\begin{tabular}{|c|c|c|c|c|c|c|}
\hline & \multicolumn{2}{|c|}{0 euro } & \multicolumn{2}{|c|}{1 cent } & \multicolumn{2}{|c|}{1 euro } \\
\hline & Congruent & Noncongruent & Congruent & Noncongruent & Congruent & Noncongruent \\
\hline \multicolumn{7}{|l|}{ Reaction time (ms) } \\
\hline Without STN-DBS & $494(18)$ & $550(18)$ & 483 (19) & $534(19)$ & 456 (19) & $517(20)$ \\
\hline With STN-DBS & 507 (19) & $562(19)$ & 488 (19) & $538(20)$ & 449 (18) & $507(20)$ \\
\hline \multicolumn{7}{|l|}{ Error rate $(\%)$} \\
\hline Without STN-DBS & $1.2(0.4)$ & $7.1(1,3)$ & $2.5(0.6)$ & $5.2(1.0)$ & $1.5(0.5)$ & $7.9(1.1)$ \\
\hline With STN-DBS & $3.3(0.6)$ & $11.2(1,9)$ & $3.0(0.6)$ & $12.8(2.0)$ & $2.6(0.6)$ & $13.1(2.4)$ \\
\hline
\end{tabular}

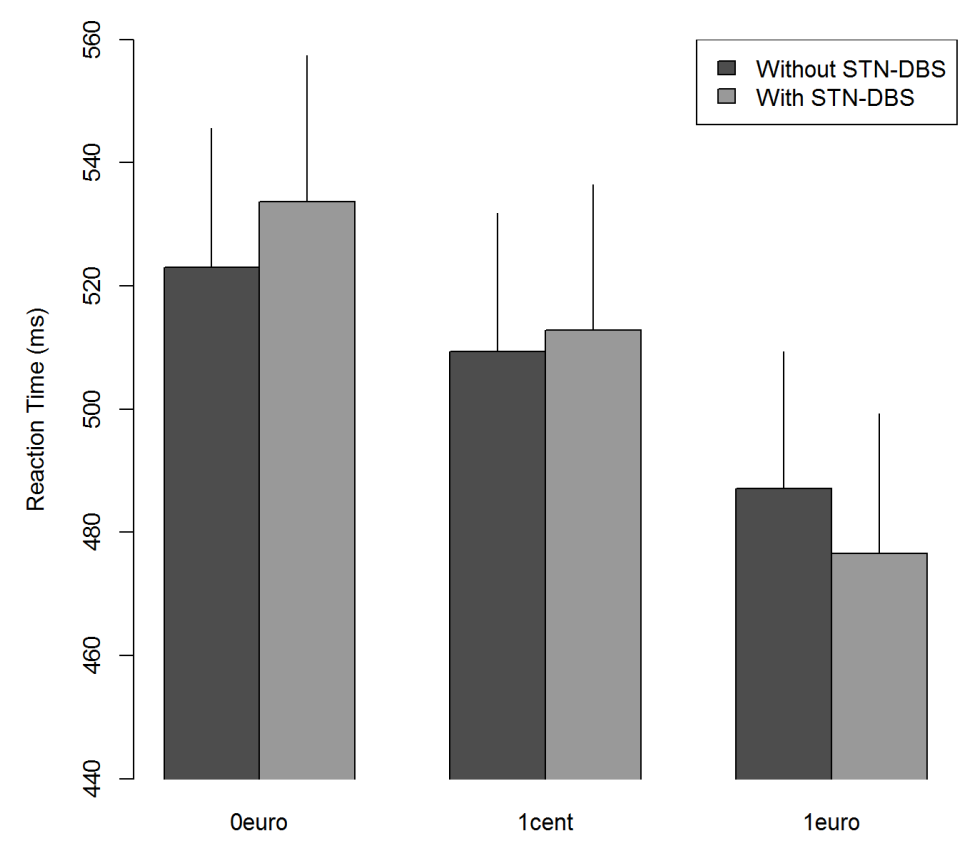

Figure 2: Reward effect, as measured by RTs for patients without or with STN-DBS. Error bars represent SEMs.

\subsubsection{Additional analyses.}

We conducted additional analyses to examine further the higher error rates for patients with STN-DBS, which occurred irrespective of experimental situation. As STN-DBS can induce impulsive decision-making (Jahanshahi et al., 2015), we analyzed error rates as a function of BIS-10 scores. We used nonparametric Spearman's rank order correlations, as the scores on 


\section{ACCEPTED MANUSCRIPT}

the questionnaire were not normally distributed. Error rates correlated significantly with scores on the BIS-10 motor impulsivity subscale (acting without thinking) in patients with STN-DBS (with an outlier: $r=0.42, p=.04$; without the outlier: $r=0.55, p=.01$ ) but not in patients without STN-DBS.

\subsubsection{Activation-suppression model.}

\subsubsection{Conditional accuracy function.}

We analyzed the effect of promised rewards on impulsive action selection by plotting response accuracy along RT, divided into three bins. First, all the participants had greater difficulty producing fast correct responses than slow ones in the noncongruent situation ( Bin $\times$ Congruence interaction: $\left.\chi^{2}=244.93, p<.0001\right)$. As expected, the greatest congruence effect was observed for the fastest responses (Bin $\times$ Congruence: first vs. second bin, $\chi^{2}=$ 86.24, $p<.0001$; second vs. third bin: $\chi^{2}=49.20, p<.0001$; first vs. third bin: $\chi^{2}=238.12, p$ $<.0001)$. Second, the promise of a reward did not change the congruence effect whatever the speed of the response $\left(\operatorname{Bin} \times\right.$ Congruence $\times$ Reward, $\left.\chi^{2}=5.26, p=.26\right)$. These results were confirmed by the analysis of the accuracy for the fastest responses (Congruence $\times$ Reward, first bin, $\chi^{2}=0.26, p=.88$ ), despite the presence of significant effects of both reward and congruence on the first bin (Reward, first bin, $\chi^{2}=11.00, p=.004$; Congruence, first bin, $\chi^{2}=$ $316.02, p<.0001)$.

Group comparisons (Figs. 3a and 3b) showed that the temporal dynamics of the congruence effect on accuracy did not differ between groups (Group $\times$ Congruence $\times$ Bin, $\chi^{2}=1.44, p=$ .49). These results were confirmed by the analysis of the accuracy for the fastest responses (Group $\times$ Congruence, first bin, $\chi^{2}=0.47, p=.49$ ). As for the influence of promised rewards on the temporal dynamics, there was no significant difference between groups (Group $\times$ 


\section{ACCEPTED MANUSCRIPT}

Reward $\times$ Congruence $\times$ Bin, $\chi^{2}=7.04, p=.13$ ). However, analysis of the fastest responses

(first bin) revealed that there was a significant Group $\times$ Congruence $\times$ Reward interaction $\left(\chi^{2}\right.$ $=6.60, p=.04)$. Patients without STN-DBS exhibited a lower congruence effect for the fastest responses in the low-incentive situation than patients with STN-DBS (Group $\times$ Congruence, first bin, 1 cent, $\chi^{2}=5.17, p=.02$; congruence effect on error rate in first bin: with STN-DBS: $€ 0=23.4 \%, 1$ cent $=24.4 \%, € 1=26.3 \%$; without STN-DBS: $€ 0=16.5 \%, 1$ cent $=10.3 \%, € 1=17.9 \%)($ Figs. $3 \mathrm{a}$ and $3 \mathrm{~b})$. In other words, patients with STN-DBS displayed greater impulsive action selection than patients without STN-DBS in the lowincentive situation. 


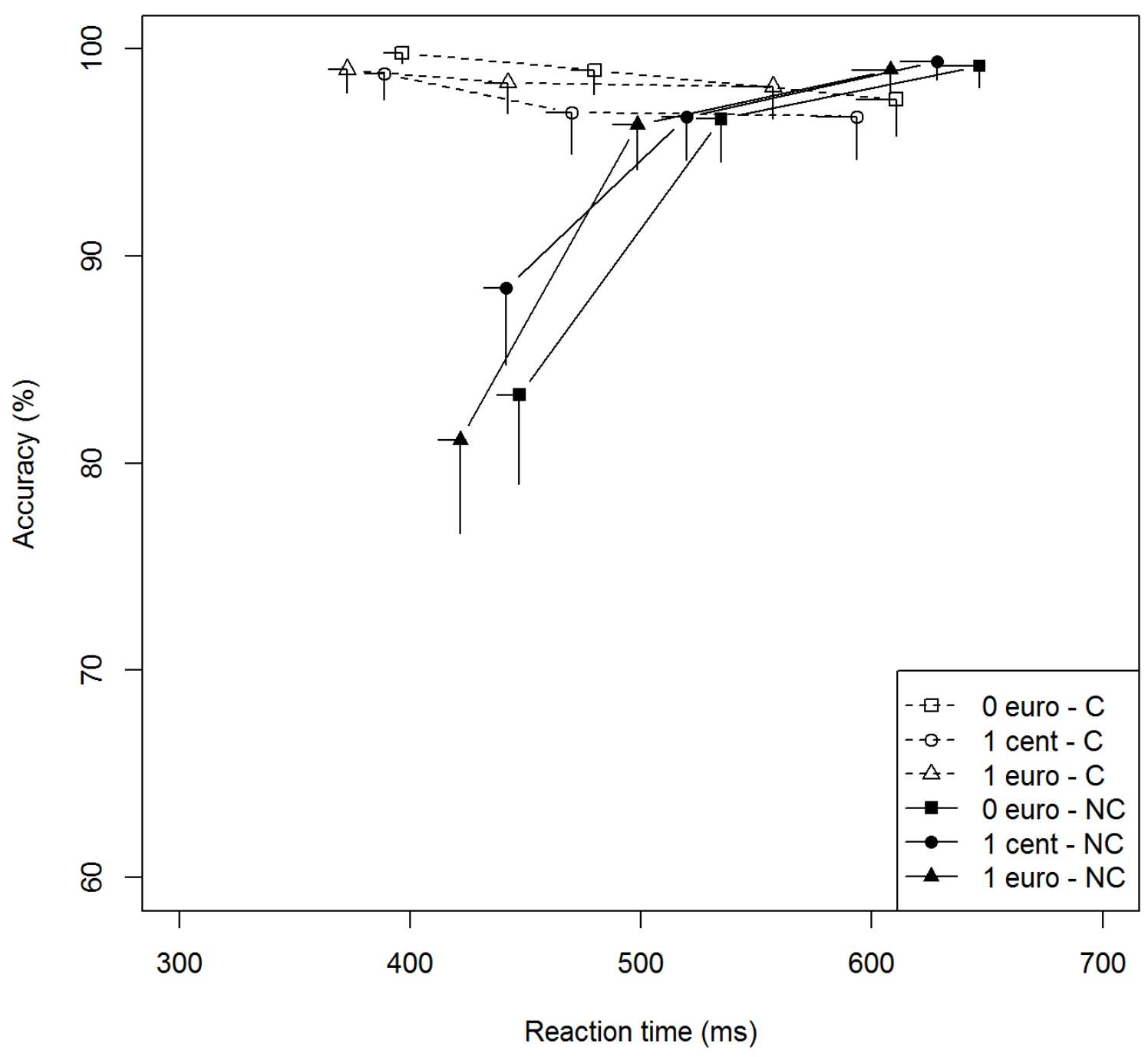

Figure 3a: Patients without STN-DBS - Conditional accuracy function for congruent and noncongruent trials according to the promised reward. Each bin represents the mean score of a third of the overall dataset. The position of each bin corresponds to the mean RT on the $x$-axis and the mean accuracy rate on the $y$-axis. Error bars represent SEMs. $\mathrm{C}=$ congruent; $\mathrm{NC}=$ noncongruent. 


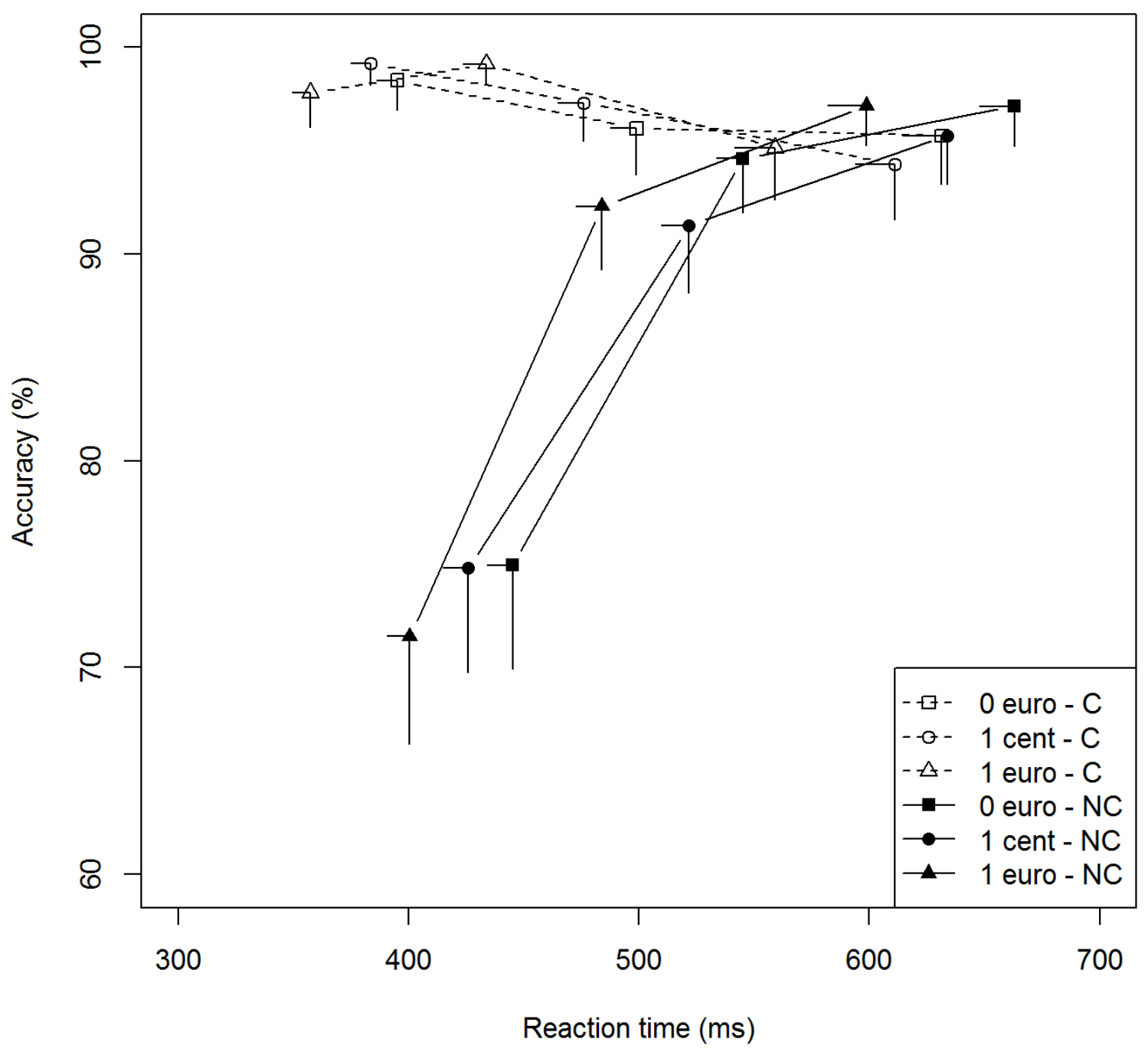

Figure 3b: Patients with STN-DBS - Conditional accuracy function for congruent and noncongruent trials according to the promised reward. Each bin represents the mean score of a third of the overall dataset. The position of each bin corresponds to the mean RT on the $x$-axis and the mean accuracy rate on the $y$-axis. Error bars represent SEMs. $\mathrm{C}=$ congruent; $\mathrm{NC}=$ noncongruent.

\subsubsection{Delta plots.}

The effects of promised rewards on the inhibitory control were analyzed by plotting the congruence effect along RT, divided into three bins, and by estimating the slope between each bin (2 slopes; Figs. 4a and 4b). 


\section{ACCEPTED MANUSCRIPT}

First, the suppression of inappropriate activations was more effective for the slowest responses in all participants, since the second slope was significantly steeper than the first slope (Slope, $\left.F_{(1,300)}=3.69, p=.05\right)$. Second, promised rewards increased the difficulty of suppressing inappropriate activations $\left(\operatorname{Reward}, F_{(2,300)}=5.05, p=.02\right)$. Compared with nonrewarded situations, the slopes were significantly weaker for the $€ 1$ situation (Reward: $€ 1$ vs. $\left.€ 0, F_{(1,200)}=3.89, p=.05\right)$ and tended towards significance for the 1 cent situation (Reward, $€ 1$ vs. 1 cent, $F_{(1,200)}=3.50, p=.06$ ), but did not differ between the $€ 0$ and 1 cent situations (Reward: $€ 0$ vs. 1 cent, $F_{(1,200)}=0.05, p=.82$ ). This effect did not depend on RTs $\left(\right.$ Reward $\times$ Slope, $\left.F_{(2,300)}=0.05, p=.82\right)$.

Group comparisons revealed no significant difference between patients with and without STN-DBS $\left(\right.$ Group, $F_{(1,300)}=0.12, p=.73$; Group $\times$ Slope, $F_{(1,300)}=.16, p=.68$; Group $\times$ Reward, $F_{(2,300)}=0.36, p=.83$; Group $\times$ Slope $\times$ Reward, $\left.F_{(2,300)}=0.97, p=.62\right)$. These results were confirmed by the comparisons for the second slope (Group, second slope, $F_{(1,150)}$ $=0.06, p=.81 ;$ Group $\times$ Reward, second slope, $\left.F_{(2,150)}=0.14, p=.93\right)$. 


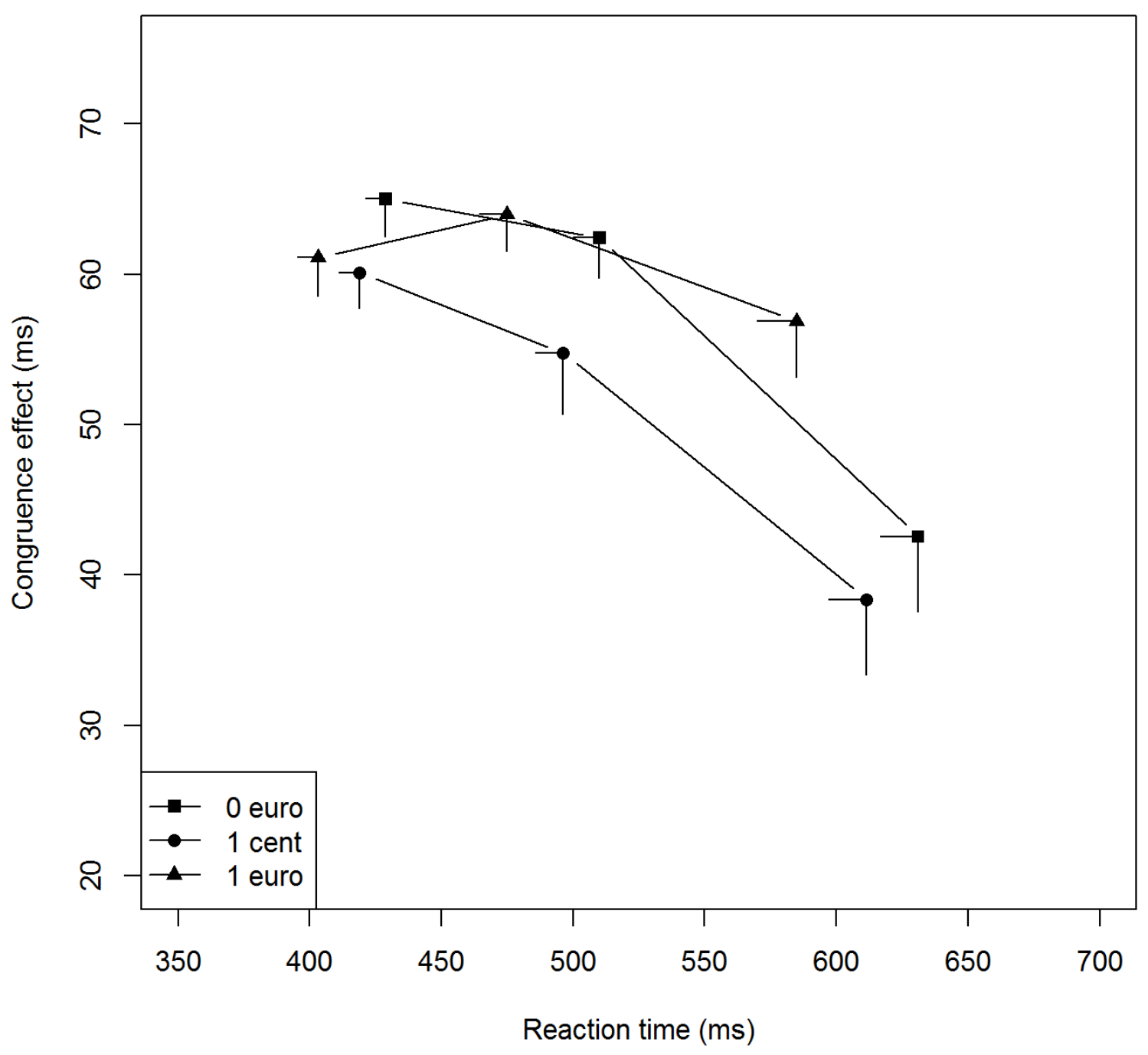

Figure 4a: Patients without STN-DBS - Delta plot of the congruence effect (noncongruent RT minus congruent RT for correct responses) according to promised reward. Each bin represents the mean score of a third of the overall dataset. The position of each bin corresponds to the mean RT on the $x$-axis and the mean congruence effect on RT on the $y$-axis. Error bars represent SEMs. 


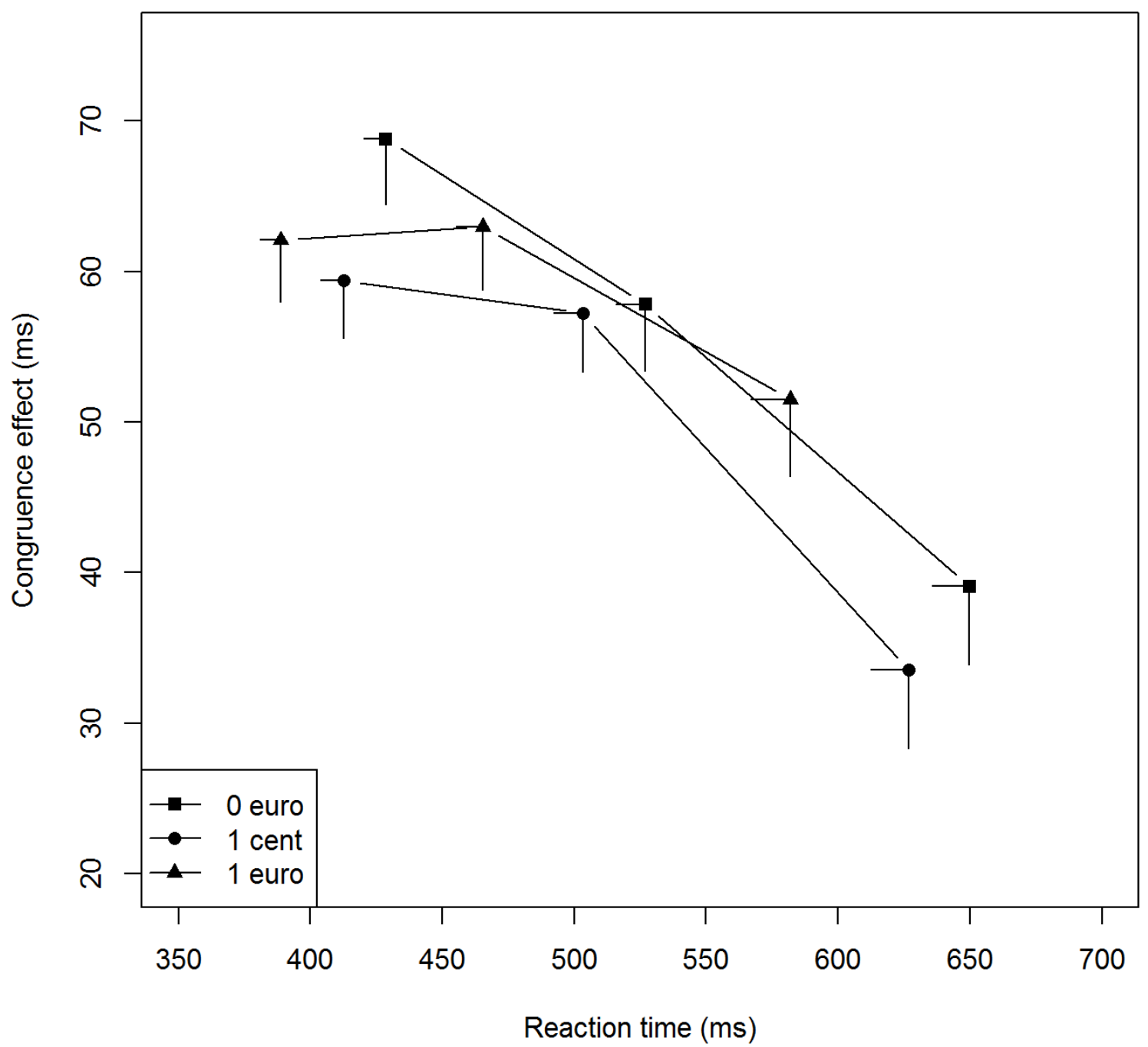

Figure 4b: patients with STN-DBS - Delta plot of the congruence effect (noncongruent RT minus congruent RT for correct responses) according to the promised reward. Each bin represents the mean score of a third of the overall dataset. The position of each bin corresponds to the mean RT on the $x$-axis and the mean congruence effect on RT on the $y$-axis. Error bars represent SEMs.

\subsection{Subgroup Analysis - PD Patients With STN-DBS}

Wylie et al. (2009) demonstrated that analyzing performances at the group level can hide the presence of pathological impulsive action selection in some individuals (Wylie et al., 2009). Accordingly, we wondered whether the patients with higher levels of impulsive action selection in the group with DBS had a specific profile in terms of clinical characteristics or 


\section{ACCEPTED MANUSCRIPT}

regarding the influence of promised rewards on the congruence effect, impulsive action selection and suppression. We therefore split the group of patients with DBS into two, according to the congruence effect on the first bin of the CAF, using a $z$ score-based criterion. We found that seven patients with DBS exhibited a congruence effect that was more than two standard deviations above the mean congruence effect calculated for the group without STNDBS.

\subsubsection{Clinical data.}

These seven patients exhibiting a high congruence effect (STN-High) only differed from the other 18 patients (STN-Low) on motor scores: the UPDRS-III score off med and off stim tended to be different, and scores with the other med/stim combinations were statistically different (Table 3). We therefore estimated the benefit of DBS on motor symptoms with the following formula: (UPDRS-III off med off stim - UPDRS-III off med with stim / UPDRS-III off med off stim. This indicated that the motor improvement following STN-DBS did not differ between the two subgroups $(p=.23)$. In sum, patients in the STN-High group had a less severe disease but derived a similar motor benefit from STN-DBS. In addition, the two groups were comparable for sociodemographic characteristics, cognitive functioning (overall cognition and executive functions), behavioral/psychiatric characteristics (anxiety, depression and apathy), time since DBS, disease duration, and medication (Table 3). Lastly, neither the mean coordinates of the selected contacts nor the mean stimulation parameters differed between the subgroups $(\mathrm{p}>.10)$. The mean coordinates of the selected contacts in the STNHigh subgroup were $13.7 \pm 1.8 \mathrm{~mm}$ lateral, $3.6 \pm 2.2 \mathrm{~mm}$ below, and $3.1 \pm 1.9 \mathrm{~mm}$ posterior to the midpoint of the AC-PC line. The mean coordinates of the selected contacts in the STNLow subgroup were $13.3 \pm 2.4 \mathrm{~mm}$ lateral, $3.1 \pm 2.5 \mathrm{~mm}$ below, and $2.0 \pm 1.8 \mathrm{~mm}$ posterior to the AC-PC line. The mean parameters for the monopolar stimulation in the STN-High 


\section{ACCEPTED MANUSCRIPT}

subgroup were $2.5 \pm 0.6 \mathrm{~V}, 60.0 \pm 0.0 \mu \mathrm{s}$, and $131.4 \pm 3.6 \mathrm{~Hz}$. The mean parameters for the monopolar stimulation in the STN-Low subgroup were $2.6 \pm 0.5 \mathrm{~V}, 60.8 \pm 5.0 \mu \mathrm{s}$, and 138.5 $\pm 19.6 \mathrm{~Hz}$. 


\section{ACCEPTED MANUSCRIPT}

Table 3: Postoperative clinical data (mean $\pm S D$ ) and comparison of the STN-High and STNLow subgroups

$\mathrm{N}$

STN-

\begin{tabular}{lcccc} 
& Low / High & STN-Low & STN-High & $p$ value \\
\hline N Men/Women & & $9 / 9$ & $3 / 4$ & 0.75 \\
Age (years) & $18 / 7$ & $58.8 \pm 6.7$ & $55.0 \pm 7.2$ & 0.25 \\
\hline Education (years) & $18 / 7$ & $10.8 \pm 4.1$ & $10.3 \pm 2.4$ & 0.95 \\
MADRS & $18 / 7$ & $4.4 \pm 5.0$ & $5.1 \pm 3.6$ & 0.33 \\
BIS-10 & $17 / 5$ & & & \\
$\quad$ Total & & $46.6 \pm 13.9$ & $47.0 \pm 11.9$ & 0.81 \\
$\quad$ Motor impulsivity & & $12.8 \pm 6.3$ & $15.4 \pm 8.0$ & 0.67 \\
$\quad$ Cognitive impulsivity & & $17.6 \pm 5.6$ & $15.2 \pm 4.6$ & 0.27 \\
$\quad$ Planning Impulsivity & $16 / 6$ & $32.4 \pm 11.5$ & $33.7 \pm 14.4$ & 0.97 \\
STAI State & $17 / 6$ & $38.6 \pm 10.8$ & $43.5 \pm 11.9$ & 0.48 \\
STAI Trait & $18 / 7$ & $29.4 \pm 8.7$ & $32.7 \pm 9.2$ & 0.50 \\
AES & $18 / 7$ & $139.7 \pm 4.6$ & $140.1 \pm 3.6$ & 0.83 \\
\hline MDRS & & & & \\
Stroop & $18 / 7$ & $71.1 \pm 10.6$ & $66.6 \pm 8.3$ & 0.27 \\
$\quad$ Color & $18 / 7$ & $98.9 \pm 16.5$ & $94.4 \pm 23.4$ & 0.58 \\
$\quad$ Word & $18 / 7$ & $42.5 \pm 9.3$ & $40.3 \pm 8.8$ & 0.56 \\
$\quad$ Color-Word & $18 / 7$ & $1.3 \pm 7.7$ & $1.1 \pm 6.8$ & 0.86
\end{tabular}

TMT

$\begin{array}{lcccr}\text { Part A } & 18 / 7 & 39.6 \pm 11.6 & 39.0 \pm 7.9 & 0.72 \\ \text { Part B } & 18 / 7 & 106.4 \pm 73.2 & 115.4 \pm 92.3 & 0.65 \\ \text { B-A } & 18 / 7 & 66.9 \pm 69.5 & 77.9 \pm 90.1 & 0.47\end{array}$

MCST

Categories

Errors

Perseverations

Verbal fluency

Semantic
Phonemic

Disease duration (years)

Time since DBS (months)

Age at disease onset (years)

Age at surgery (years)

UPDRS-III on med - on stim

UPDRS-III on med - on stim

UPDRS-III on med - off stim

UPDRS-III off med - on stim

UPDRS-III off med - off stim

Schwab and England (\%) "on"

Schwab and England (\%) "off"

Hoehn and Yahr on med
$18 / 7$

$18 / 7$

$18 / 7$

$$
5.5 \pm 1.1
$$

$5.1 \pm 1.7$

0.66

$6.6 \pm 6.9$

$8.6 \pm 9.7$

0.88

$2.3 \pm 3.3$

$3.1 \pm 2.7$

0.22

$\begin{array}{lccc}18 / 7 & 28.7 \pm 8.2 & 25.3 \pm 6.7 & 0.50 \\ 18 / 7 & 20.7 \pm 6.5 & 20.3 \pm 6.0 & 0.98 \\ 18 / 7 & 12.7 \pm 2.0 & 12.7 \pm 4.7 & 0.65 \\ 18 / 7 & 29.9 \pm 23.9 & 30.4 \pm 31.1 & 0.95 \\ 18 / 7 & 46.5 \pm 6.9 & 42.9 \pm 7.3 & 0.26 \\ 18 / 7 & 56.7 \pm 7.1 & 53.0 \pm 7.3 & 0.22 \\ 18 / 7 & 10.6 \pm 4.9 & 5.5 \pm 5.1 & 0.04 \\ 18 / 7 & 10.6 \pm 4.9 & 5.5 \pm 5.1 & 0.04 \\ 18 / 7 & 18.6 \pm 8.7 & 9.8 \pm 4.7 & 0.02 \\ 18 / 7 & 24.9 \pm 11.2 & 13.4 \pm 6.3 & 0.03 \\ 18 / 7 & 40.5 \pm 12.0 & 30.9 \pm 9.0 & 0.08 \\ 18 / 7 & 92.2 \pm 8.8 & 88.6 \pm 3.8 & 0.23 \\ 18 / 7 & 82.2 \pm 10.0 & 77.1 \pm 17.0 & 0.80 \\ 18 / 7 & 1.0 \pm 1.0 & 1.3 \pm 1.1 & 0.52\end{array}$




\section{ACCEPTED MANUSCRIPT}

$\begin{array}{lcccc}\text { Hoehn and Yahr off med } & 18 / 7 & 2.3 \pm 0.8 & 1.6 \pm 1.3 & 0.45 \\ \text { LEDD (mg/day) } & 18 / 7 & 785.5 \pm 435.0 & 1044.7 \pm 490.0 & 0.18\end{array}$

Note. MADRS = Montgomery and Asberg Depressive Rating Scale; BIS-10 = Barrat Impulsiveness Scale; STAI

$=$ State-Trait Anxiety Inventory; AES = Apathy Evaluation Scale; MDRS = Mattis Dementia Rating Scale; TMT

$=$ Trail Making Test MCST $=$ Modified Wisconsin Card Sorting Test; UPDRS $=$ Unified Parkinson's Disease

Rating Scale; LEDD = levodopa equivalent daily dose. p.value were estimated by using Mann-Whitney U test

\subsubsection{Overall RT and accuracy.}

Subgroups did not differ on overall RT (Subgroup, $F_{(1,24)}=0.19, p=.66$ ) but did on overall accuracy (Subgroup, $\chi^{2}=8.15, p<.0001$ ). The STN-High group produced more erroneous responses (accuracy: STN-High $=14.5 \% ; \mathrm{STN}-\mathrm{Low}=5.0 \%)$. The congruence effect was greater in STN-High patients (Subgroup $\times$ Congruence, RT, $F_{(1,8206)}=18.35, p<.0001$; accuracy, $\left.\chi^{2}=15.40, p<.0001\right)$. The reduction in $\mathrm{RT}$ with the promise of a reward was greater in the STN-High subgroup (Subgroup $\times$ Reward, RT, $F_{(2,8206)}=6.12, p=.05$; accuracy, $\chi^{2}=0.25, p=.88$ ). The STN-High subgroup produced faster responses in the high reward situation than in the low one (Subgroup $\times$ Reward, RT: $€ 1$ vs. 1 cent, $F_{(2,11094)}=5.82$, $p=.02 ; € 1$ vs. $€ 0, F_{(1,5480)}=0.87, p=.35 ; 1$ cent vs. $\left.€ 0, F_{(1,5468)}=2.29, p=.13\right)$. The influence of promised rewards on the congruence effect, as reflected by accuracy, also differed between subgroups (Subgroup $\times$ Congruence $\times$ Reward, RT, $F_{(2,8206)}=3.56, p=.17$; accuracy, $\chi^{2}=9.66, p=.008$ ) (Fig. 5). Promised reward only modulated the congruence effect in the STN-High subgroup (Congruence $\times$ Reward, accuracy: STN-High, $\chi^{2}=8.69, p=.008$; STN-Low, $\left.\chi^{2}=2.70, p=.26\right)$. The congruence effect was greater in the $€ 1$ situation in the STN-High subgroup (Congruence $\times$ Reward, accuracy: $€ 0$ vs. 1 cent, $\chi^{2}=0.01, p=.90 ; € 0$ vs. $€ 1, \chi^{2}=6.85, p=.009 ; 1$ cent vs. $€ 1, \chi^{2}=7.61, p=.006$ ) (Congruence effect: $€ 0=17.3 \% ; 1$ cent $=18.3 \% ; € 1=25.9 \%)$. 


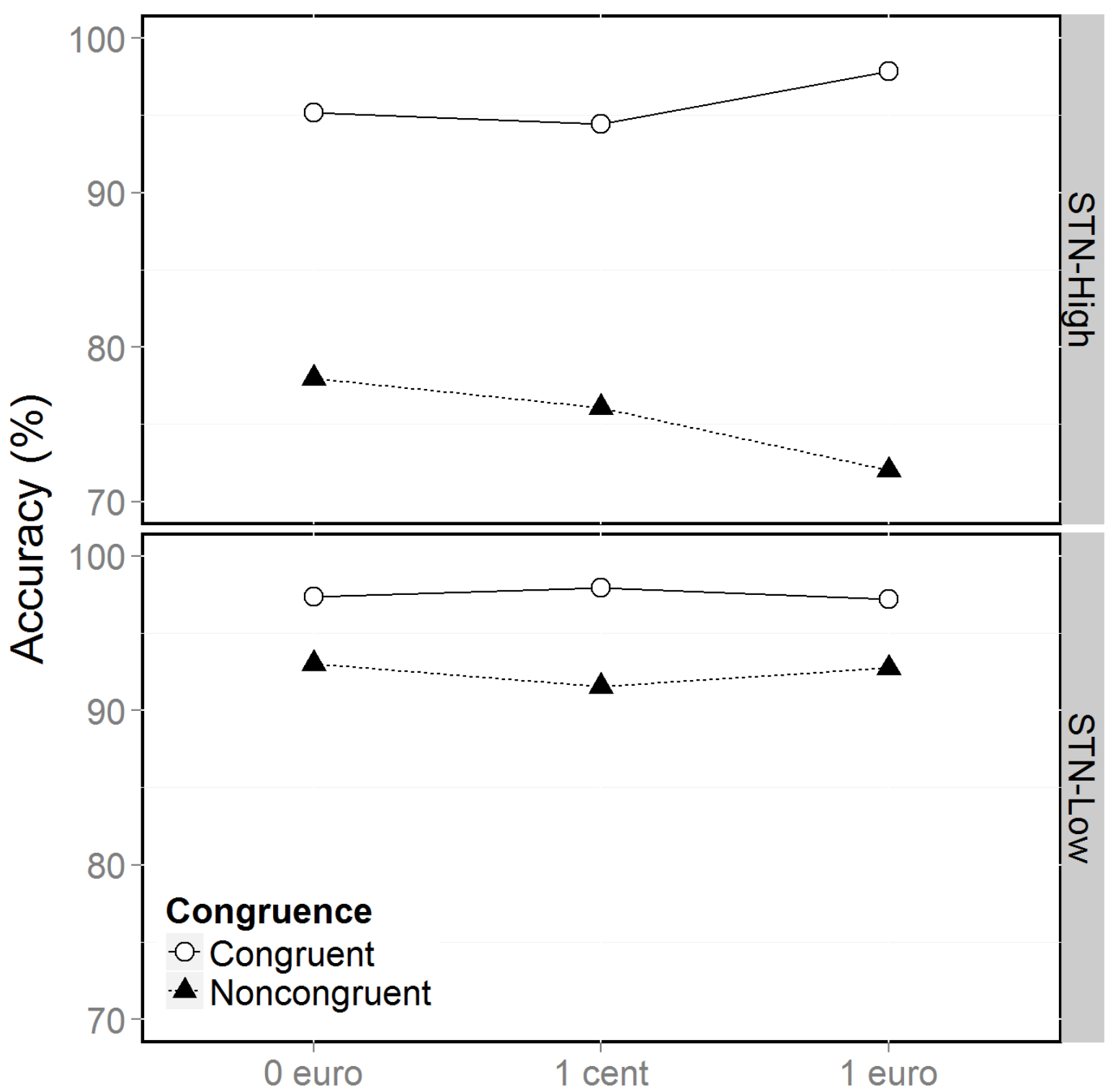

Figure 5: Accuracy $(\%)$ as a function of congruence and reward in the STN-High (top) and STN-Low (bottom) subgroups.

\subsubsection{Activation-suppression model.}

\subsubsection{Conditional accuracy function.}

We analyzed the effect of promised rewards on impulsive action selection by plotting response accuracy along $\mathrm{RT}$, divided into three bins.

A subgroup comparison showed that the temporal dynamics of the congruence effect on accuracy differed between subgroups (Subgroup $\times$ Congruence $\times \operatorname{Bin}, \chi^{2}=6.53, p=.04$ ) 


\section{ACCEPTED MANUSCRIPT}

(Figs. 6a and 6b). These results were confirmed by analysis of the error rate for the fastest responses, which revealed a greater congruence effect for the STN-High subgroup (Subgroup $\times$ Congruence, first bin, $\chi^{2}=13.58, p=.0002$ ). Regarding the influence of promised reward on the temporal dynamics, there was no significant difference between the two subgroups $\left(\right.$ Subgroup $\times$ Reward $\times$ Congruence $\times$ Bin, $\left.\chi^{2}=0.89, p=.93\right)$. Once again, these results were confirmed by the analysis of the accuracy for the fastest responses (Subgroup $\times$ Congruence $\times$ Reward, $\left.\chi^{2}=2.68, p=.26\right)$. 


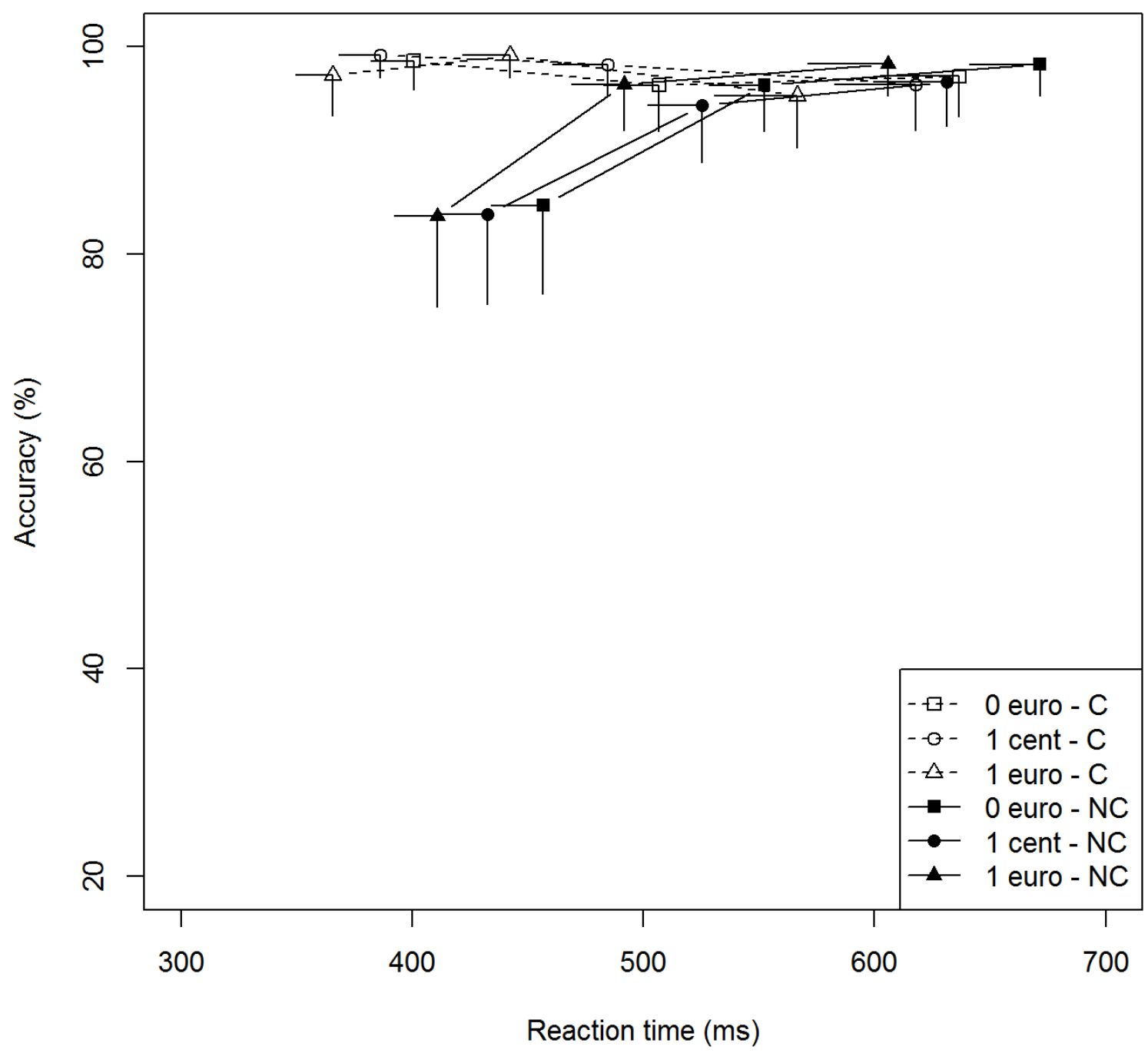

Figure 6a: Conditional accuracy function for congruent and noncongruent trials according to promised rewards in the STN-Low subgroup. Each bin represents the mean score of a third of the overall dataset. The position of each bin corresponds to the mean RT on the $x$-axis and the mean accuracy rate on the y-axis. Error bars represent SEMs. $\mathrm{C}=$ congruent $\mathrm{NC}=$ noncongruent. 


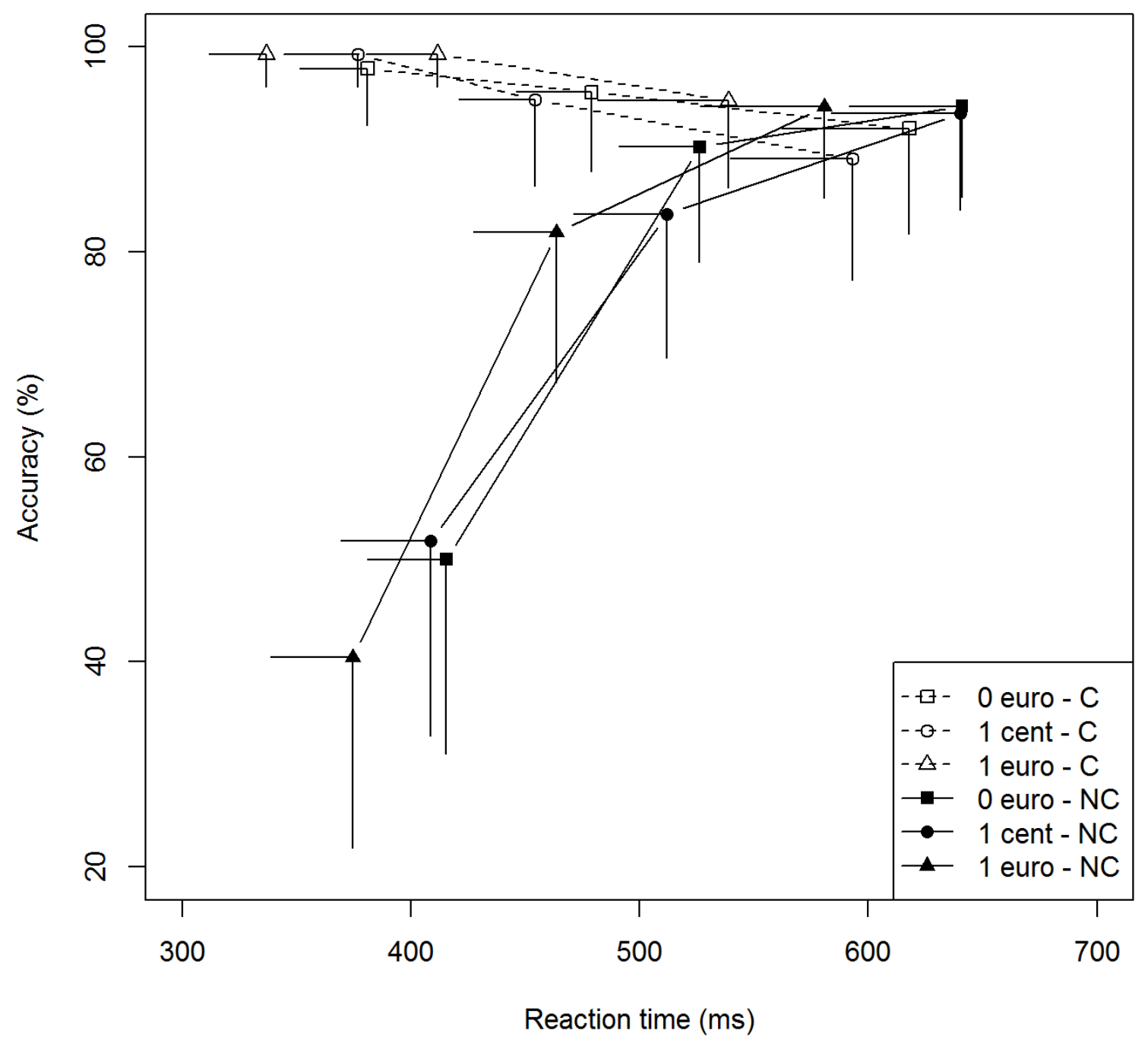

Figure 6b: Conditional accuracy function for congruent and noncongruent trials according to promised rewards in the STN-High subgroup. Each bin represents the mean score of a third of the overall dataset. The position of each bin corresponds to the mean RT on the $x$-axis and the mean accuracy rate on the $y$-axis. Error bars represent SEMs. $\mathrm{C}=$ congruent; $\mathrm{NC}=$ noncongruent

\subsubsection{Delta plots.}

We analyzed the effects of the promised rewards on impulse suppression by plotting the congruence effect along RT, divided into three bins, and by estimating the slope between each bin (two slopes). 


\section{ACCEPTED MANUSCRIPT}

The slopes differed between the two subgroups (Subgroup $\times$ Slope, $F_{(1,150)}=4.10, p=.04$;

Figs. 7a and 7b). Only the STN-High subgroup showed a significant difference between slopes (Slope, STN-Low, $F_{(1,108)}=0.03, p=.87$; STN-High, $F_{(1,42)}=3.81, p=.05$ ). This effect indicated that selective suppression needed more time to become effective in the STNHigh subgroup $(m$ first slope $=0.06 ; m$ second slope $=-0.15$ ) than in the STN-Low subgroup ( $m$ first slope $=-0.07 ; m$ second slope $=-0.11)$.

No other effect was observed (Subgroup, $F_{(1,24)}=0.00, p=.98 ;$ Subgroup $\times$ Reward, $F_{(2,150)}$ $=3.08, p=.21 ;$ Subgroup $\times$ Slope $\times$ Reward, $\left.F_{(2,150)}=0.85, p=.65\right)$, and the comparison of the last slope was nonsignificant (Subgroup, second slope, $F_{(1,24)}=1.34, p=.25$; Subgroup $\times$ Reward, second slope, $\left.F_{(2,75)}=0.79, p=.67\right)$. 


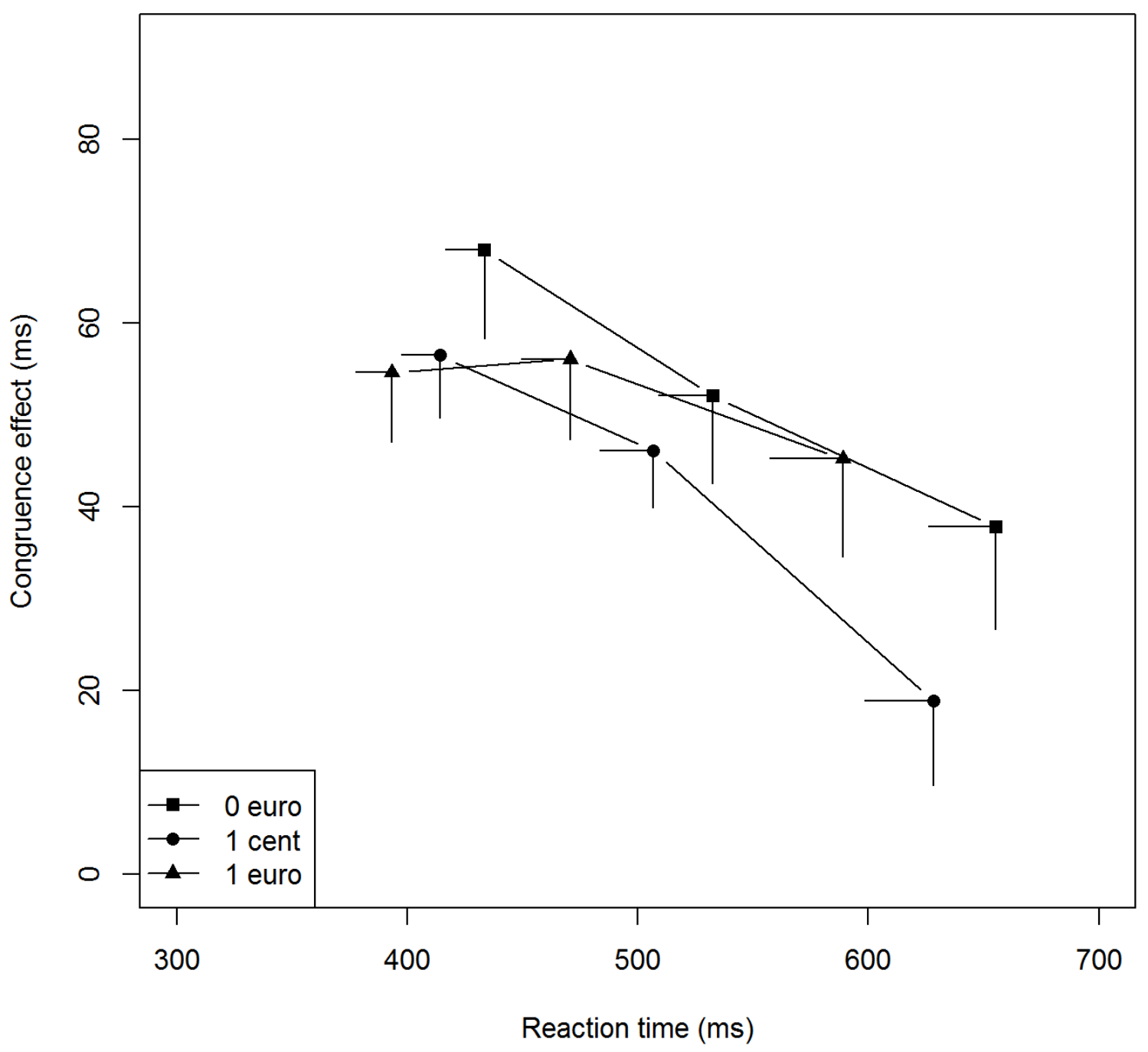

Figure 7a: Delta plot of the congruence effect (noncongruent RT minus congruent RT for correct responses) according to promised rewards in the STN-Low subgroup. Each bin represents the mean score of a third of the overall dataset. The position of each bin corresponds to the mean RT on the $x$-axis and the mean congruence effect on RT on the $y$-axis. Error bars represent SEMs. 


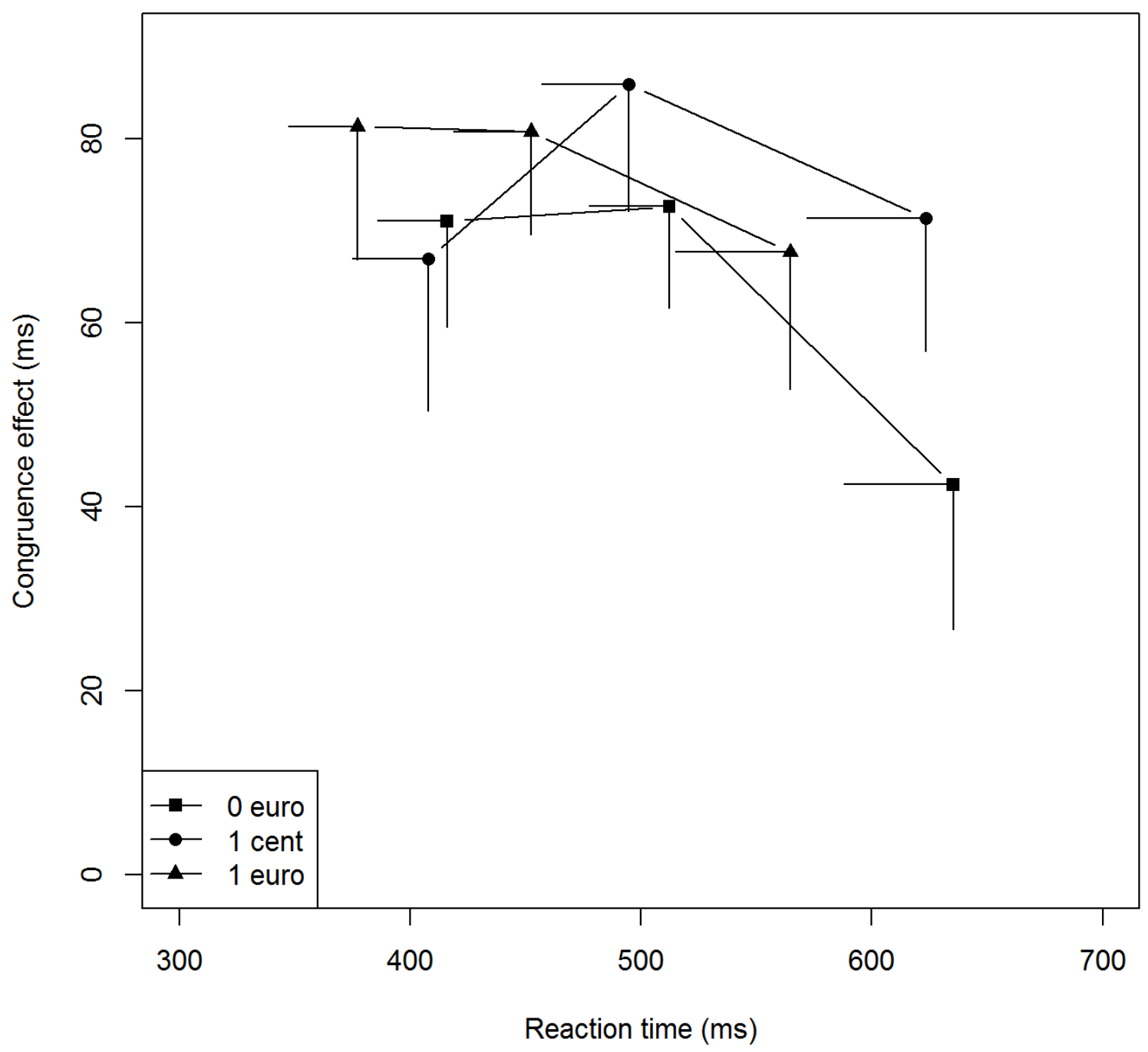

Figure 7b: Delta plot of the congruence effect (noncongruent RT minus congruent RT for correct responses) according to promised rewards in the STN-High subgroup. Each bin represents the mean score of a third of the overall dataset. The position of each bin corresponds to the mean RT on the $x$-axis and the mean congruence effect on RT on the $y$-axis. Error bars represent SEMs.

\section{DISCUSSION}

The current study investigated the influence of STN-DBS on cognitive action control in an incentive context. To this end, we administered a rewarded Simon Task to 50 patients with 


\section{ACCEPTED MANUSCRIPT}

PD, half of whom were being treated with STN-DBS. First, we compared these two groups according to the dual-route model, to assess the cognitive effort needed to resolve conflict situations. Second, we conducted further analyses based on the activation-suppression model (Ridderinkhof, 2002a, 2002b, 2004), to investigate impulse selection and suppression. Third, we compared the congruence effect and impulse selection and suppression in patients undergoing STN-DBS, split into two subgroups according to the presence or absence of pathological impulsive action selection.

\subsection{Group Comparisons: Overall Cohort}

\subsubsection{Clinical information.}

The two groups of patients with PD (with and without STN-DBS) did not differ on sociodemographic data (sex, age and education level), disease characteristics (disease duration and severity of motor symptoms), cognitive abilities, or psychiatric aspects (anxiety, impulsivity, apathy and depression). Thus, the only difference between the two groups was the treatment used to improve their motor symptoms.

\subsubsection{Dual-route model.}

As expected, all participants had greater difficulty producing correct responses in the noncongruent situation than in the congruent one, thus exhibiting the classic congruence effect, as reflected by both RT and accuracy. In addition, and as previously reported (Padmala and Pessoa, 2011), the promise of a reward increased the speed of responses in relation to the size of the reward, regardless of the congruence situation. This suggests that promised rewards have the potential to increase time pressure. Promised rewards also increased the 


\section{ACCEPTED MANUSCRIPT}

congruence effect, as the difficulty participants had producing correct responses in the noncongruent versus congruent situation was heightened in the high-incentive context. This result fits in well with previous studies investigating the influence of speed-accuracy strategy on cognitive action control. These studies showed that there is a greater congruence effect in the Simon task when the instructions emphasize speed rather than accuracy in responding (van Wouwe et al., 2014). We interpreted the negative impact of promised rewards on cognitive action control as the consequence of the pressure induced by the difficulty of the task. Thus, promised rewards increased time pressure and, in turn, made conflict resolution harder. As the full reward was only obtained for the fastest correct responses (one sixth less than the mean baseline RT), we postulated that displaying the reward at the beginning of the trial increased the time pressure, especially in the most highly rewarded situation, which in turn made conflict resolution harder. This assumption fits in with the choking under pressure model, which states that our performances deteriorate when, under excessive pressure of incentive motivation, we try but fail to do our best (Baumeister, 1984; Beilock et al., 2004; Mobbs et al., 2009).

The comparison between patients with STN-DBS and patients without STN-DBS allowed us to establish the precise influence of STN-DBS on these processes.

First, compared with the nonimplanted patients, the patients with STN-DBS produced more erroneous responses, irrespective of both congruence and reward. By contrast, the two groups did not differ on overall RT. Therefore, as suggested previously (Hershey et al., 2010), the higher error rate was unlikely to be due to a modulation of the speed/accuracy trade-off. Instead, it probably reflected the fact that performing a cognitive task requiring high cognitive control was harder for the patients treated with STN-DBS. In addition, the error rate correlated positively with scores on the BIS-10 motor impulsivity subscale. Therefore, the 


\section{ACCEPTED MANUSCRIPT}

patients with STN-DBS who acted without thinking produced more erroneous responses whatever the situation. This correlation was specific to patients with STN-DBS, even though patients with and without DBS had similar total BIS-10 scores. These results complement those of a previous study that had shown that STN-DBS can increase the expression of trait impulsivity in daily life, as measured with the BIS-10 (Hälbig et al., 2009). The current study showed for the first time that trait impulsivity correlates with STN-DBS-induced decrease in performances on an experimental task. One attractive hypothesis is that STN-DBS liberates the expression of motor impulsivity, possibly characterized by the production of more erroneous responses during a task requiring high cognitive control.

Second, there was a greater increase in speed according to promised reward in patients with versus without DBS. Thus, promised rewards facilitated action preparation, and this effect was greater with STN-DBS. This is in line with recent studies showing that STN-DBS can change the way reward information is processed (Palminteri et al., 2013; Wagenbreth et al., 2015). Compared with an inactive stimulation condition, activation of DBS has been reported to allow patients to produce more effort (squeezing a handgrip) in order to gain promised rewards (Palminteri et al., 2013). This suggests that STN stimulation could boost the incentive effect of promised rewards. In another study using a rewarded Go No Go-like task, activation of STN-DBS stimulation was also shown to facilitate Go responses more strongly in a rewarded situation than in a punished one (Wagenbreth et al., 2015). Taken together, these results suggest that STN-DBS amplifies the facilitation of action selection in incentive context, or more generally, that it favors the production of incentive actions. However, further studies comparing patients on versus off stimulation are needed to better characterize the influence of STN inhibition on these processes.

Finally, group comparisons showed that neither the congruence effect, which represents the cognitive effort needed to resolve conflict situations, nor the influence of promised reward on 


\section{ACCEPTED MANUSCRIPT}

the congruence effect, was impacted by STN-DBS. The lack of a significant difference between groups concerning the size of the congruence effect suggests that the resolution of conflict situations was no more difficult for patients with STN-DBS than for patients without STN-DBS. Then again, analyzing the overall dataset may not be appropriate to detect subtle behavioral differences between patients with and without STN-DBS, as discussed by Wylie, Ridderinkhof, Elias, et coll., (2010). Similarly, the lack of significant group differences concerning the modulation of the congruence effect by promised rewards could be the consequence of the low power of analyses conducted on the overall dataset.

As a consequence, we conducted complementary analyses based on the activation-suppression model to better investigate the role of the STN in cognitive action control depending on the incentive context.

\subsubsection{Activation-suppression model.}

The activation-suppression model is useful for investigating impulsive action selection and suppression (Ridderinkhof, 2002a, 2002b, 2004). The cognitive processes involved in conflict resolution are dynamic and change over time. The fastest actions are the most impulsive ones, as they are driven by the irrelevant stimulus information, leading to more erroneous responses in noncongruent situations. Accordingly, we observed higher error rates for fast responses in noncongruent situations in both groups. Conversely, slower actions are less influenced by the inappropriate activation driven by the irrelevant stimulus information. The slower the response, the more time there is to activate the selective inhibition of the irrelevant response tendency. Accordingly, the delta plots showed that the congruence effect (measured with the RTs for correct responses) was weakest for the slowest responses. However, a group comparison performed on the whole sample of patients failed to replicate previous results showing that STN stimulation leads to more impulsive action selection and more efficient impulse suppression (Wylie et al., 2010b). Possible explanations for this discrepancy are 


\section{ACCEPTED MANUSCRIPT}

differences in the characteristics of the patient population and the study design. Despite a similar disease duration, our patients with STN-DBS had a more severe disease than those in the study by Wylie, Ridderinkhof, Elias et al., (2010). In addition, these authors compared the same patients in two stimulator activation conditions, thus reducing the interpatient variability of group comparisons. Finally, they benefited from finer resolution in the RT distribution, allowing for a better estimation of impulse selection and suppression. In their study, the temporal dynamics were analyzed after dividing RTs into seven bins, whereas we divided RTs into three, owing to the higher number of experimental conditions in our study. Although our task contained no fewer than 360 trials, because we had three different reward conditions, we had to restrict the number of bins in order to maintain a satisfactory number (20) of trials in each one (Wylie, Ridderinkhof, Elias et al., 2010). Inflating the duration of the task by adding more trials per condition would probably have been deleterious to the patients with PD, because of their attentional deficit.

We nevertheless observed that the influence of promised reward on impulsive action selection differed between the two groups. Promised reward only modulated the congruence effect in the first bin in patients without STN-DBS. This group difference mainly stemmed from the lower congruence effect in the 1 cent situation in patients without STN-DBS. We observed a beneficial effect of low promised reward on these patients, as they produced fewer errors in this situation, whereas the patients with STN-DBS derived no benefit from it. Additional studies are needed to replicate these results and fully understand this specific reduction in impulsive action selection in a low-incentive context in patients with medically treated PD. For the time being, we can simply state that the influence of promised reward on impulsive action selection seems to differ between patients with and without STN-DBS. Factors other than STN-DBS may have contributed to these results, but their influence was probably only limited. The two groups did not differed on the demographic, disease characteristics, 


\section{ACCEPTED MANUSCRIPT}

cognitive and psychiatric abilities, and dopaminergic treatment variable, which we included in our statistical analyses, did not significantly influenced the results.

Last, action suppression was less effective in an incentive context: promised rewards increased the difficulty of suppressing inappropriate activation (delta plot slopes). There was no difference between groups, suggesting that this interaction was not strongly influenced by STN-DBS.

\subsection{Subgroup Comparisons Within the STN-DBS Cohort}

As previous studies had shown that the pathological selection of impulsive actions concerns only a subgroup of PD patients (Wylie et al., 2009; Wylie, Ridderinkhof, Bashore, \& van den Wildenberg, 2010), and as our own data pointed to the presence of just such a subgroup among the patients with STN-DBS, we decided to split this group according to whether or not they displayed pathological impulsive action selection. To this end, we compared the congruence effect on accuracy for the fastest responses (first bin in the CAF) for each implanted patient with the corresponding mean congruence effect in patients without STNDBS. By so doing, we found that 7 of the 25 patients with STN-DBS exhibited pathological impulsive action selection (STN-High subgroup) and 18 behaved similarly to patients without STN-DBS (STN-Low subgroup). We then compared these two subgroups on clinical data and task performances, in the light of both the dual-route model and the activation-suppression model (Ridderinkhof, 2002a, 2002b, 2004).

\subsubsection{Clinical information.}

The seven patients in the STN-High subgroup differed from the STN-Low subgroup only in terms of motor symptom severity. Impulsive patients had less severe disease, as shown by the UPDRS-III scores, which were either significant or tended toward significance. Importantly, 


\section{ACCEPTED MANUSCRIPT}

other disease characteristics, including disease duration, motor improvement brought about by STN-DBS, LEDD and time since surgery did not differ between subgroups. Furthermore, the two subgroups did not differ on sociodemographic data (sex, age and education level), cognitive abilities, or psychiatric disorders (anxiety, impulsivity, apathy and depression). These results suggest that implanted patients who exhibited the highest impulsive action selection were those with the lowest disease severity at the time of the assessment. One attractive hypothesis is that STN-DBS induces cognitive side effects such as impulsive action selection in patients with less severe symptoms. This is a very important point, as STN-DBS is now indicated in the early stages of the disease (Schuepbach et al., 2013), and suggests that such patients should undergo careful clinical monitoring following STN-DBS for the occurrence or increase in impulsivity.

\subsubsection{Dual-route model.}

As the two subgroups were formed according to the congruence effect for the fastest responses, which was largely corroborated by an increased error rate in the incongruent situation, it is not surprising that the STN-High subgroup produced more erroneous responses, irrespective of experimental situation, and that the congruence effect was greater for both accuracy and RT in this subgroup. Subgroup comparisons further indicated that STN-High and STN-Low patients differed on the influence of promised reward on the effort needed to resolve conflict situations. Promised reward only modulated the congruence effect, as measured by the error rate, in the STN-High subgroup. These patients had the greatest difficulty producing correct responses in the highest incentive context. Interestingly, this greater congruence effect manifested itself in the conjunction of a decrease in the error rate in the congruent situation and an increase in the error rate in the noncongruent situation. This suggests that irrelevant stimulus information had a stronger influence on response selection in 


\section{ACCEPTED MANUSCRIPT}

the higher incentive context. This stronger influence was beneficial when the relevant and irrelevant stimulus information activated the same motor program (congruent situation), but deleterious when the relevant and irrelevant information activated different motor ones (noncongruent situation). In sum, these results show that an incentive context modulates the influence of irrelevant information on action selection in STN-High. In other words, an incentive context has a more deleterious effect on the ability to resolve a conflict in the most impulsive patients with PD undergoing stimulation.

\subsubsection{Activation-suppression model.}

Analysis of the accuracy of the fastest responses showed that seven implanted patients displayed a greater congruence effect than the patients without STN-DBS. Fast responses were therefore captured more by the automatic stimulus-response associations in more than a quarter of patients with STN-DBS, and this pathological selection of impulsive actions led to more erroneous responses in conflict situations. This result is in line with the suggestion that the STN plays a role in impulsive action selection (Jahanshahi et al., 2015; Ridderinkhof et al., 2011; Wylie, Ridderinkhof, Elias et al., 2010; Zavala et al., 2015). According to recent models, the STN quickly blocks all action tendencies in conflict situations, and continues to do so until the conflicts are resolved, thus preventing impulsive responses. Disturbance of STN activity by DBS therefore hinders this function, allowing for the emergence of impulsive responses. The fact that the most impulsive patients in our study had the least severe disease raises several intriguing hypotheses. One is that STN-DBS patients with more severe disease have developed compensatory mechanisms that prevent impulsive responses, unlike patients with less severe disease. 


\section{ACCEPTED MANUSCRIPT}

Furthermore, in the STN-High subgroup, the greatest congruence effect in a reward context was not reflected in RT, as shown by the lack of a significant interaction between group, bin, congruence and reward situation.

Finally, the functioning of the inhibitory processes allowing for the suppression of the inappropriate activation triggered by irrelevant stimulus information differed between the two subgroups. STN-High patients needed more time to efficiently suppress inappropriate activations, whereas selective suppression was active from the outset in STN-Low patients. This difference probably stemmed from greater response capture in the STN-High subgroup which, in turn, made the responses harder to suppress, especially the fastest responses that were more strongly captured (Ridderinkhof, 2002a, 2002b, 2004).

\subsection{Limitations and further directions.}

Several points have to be considered when interpreting our results. First, the two groups of patients (with and without DBS) may have differed on some aspects, even though we used a matched pairs design, and group comparisons did not reveal any significant differences on cognitive, behavioral/psychiatric, or motor characteristics. We chose to use an intergroup design in this study, even though comparing the same patients before and after surgery (intragroup design) would have avoided the problem of intergroup variability. This type of experimental design would provide a useful means of confirming our results. Second, the comparison between the STN-High and STN-Low subgroups failed to reveal any significant difference in either cognitive, behavioral/psychiatric or motor characteristics, coordinates of selected contacts, or stimulation parameters. We did, however, find that patients who were more prone to impulsive action selection had less severe motor symptoms. This result suggests that patients with a less severe disease are more at risk of developing impulsive 


\section{ACCEPTED MANUSCRIPT}

action selection following surgery. However, this result needs to be confirmed by longitudinal studies investigating the same patients before and after surgery, with a special focus on preoperative disease severity. This type of approach would make it possible to identify groups of patients who are more at risk of developing a postoperative cognitive action control deficit, notably in an incentive context. Third, the respective roles of the various aspects of stimulation still have to be clarified. We investigated the overall influence of STN-DBS, which can entail a reduction in dopaminergic treatment, lesions and electric stimulation effects. Conclusions regarding each of these aspects are therefore limited. Dopaminergic treatment may well modulate cognitive action control in an incentive context (Aarts et al., 2014). However, we cannot draw any conclusions about this point, for whereas task performances differed across groups, as we did not observe any differential influence of medication on these performances, either between patients with and without STN-DBS or between the STN-High and STN-Low subgroups. This point could be clarified by assessing the patients in all four treatment conditions (On/Off $\mathrm{x}$ DBS and medication), although this would generate limitations in terms of motor discomfort and, above all, attention deficit. Fourth, the link between cognitive action control in an incentive context and impulse control disorders (ICDs) needs to be investigated. The current study could not clarify this point because we did not include patients with ICDs. Further research with such patients would be highly relevant.

\subsection{Conclusion.}

The current study yielded a great deal of information regarding the influence of STN-DBS on cognitive action control processes during conflict resolution in different incentive contexts. First, motor impulsivity, as measured with the BIS-10, only correlated with the error rate in 


\section{ACCEPTED MANUSCRIPT}

patients with PD undergoing STN-DBS, suggesting that STN-DBS favors the expression of motor impulsivity. Second, the promise of a reward increased response speed more in STNDBS patients, suggesting that STN-DBS boosts the incentive effect of promised rewards. Third, STN-DBS may modulate the influence of promised reward on conflict resolution, as implanted patients exhibited more impulsive action selection in the low-incentive context. Fourth, although STN-DBS did not systematically induce impulsive action selection in patients, those with a less severe disease seemed to be at greater risk of developing impulsive behaviors. This information could be of importance, by alerting physicians to the potential risk of postoperative impulsivity in patients with less severe PD. Fifth, the implanted PD patients who displayed the most impulsive action selection had the greatest difficulty resolving conflict when exposed to an incentive context. In summary, as a result of the combined effects of electrical stimulation and the lesions potentially induced by electrode implantation, STN-DBS affects the cognitive processes involved in conflict resolution, reward processing, and probably also the influence of promised rewards on conflict resolution in patients with PD. Further studies using on versus off stimulation designs or local field potential recordings could help us to understand the role played by the STN in the interaction between incentive context and cognitive action control. 


\section{ACCEPTED MANUSCRIPT}

\section{Acknowledgements}

The study was funded by University of Rennes 1 .

\section{BIBLIOGRAPHY}

Aarts, E., Wallace, D.L., Dang, L.C., Jagust, W.J., Cools, R., D’Esposito, M., 2014. Dopamine and the cognitive downside of a promised bonus. Psychol. Sci. 25, 1003-1009. doi:10.1177/0956797613517240

Bates, D., Maechler, M., Bolker, B., Walker, S., Christensen, R.H.B., Singmann, H., Dai, B., Grothendieck, G., 2015. Ime4: Linear mixed-effects models using “Eigen” and S4.

Baumeister, R.F., 1984. Choking under pressure: self-consciousness and paradoxical effects of incentives on skillful performance. J. Pers. Soc. Psychol. 46, 610-620.

Baylé, F.J., Bourdel, M.C., Caci, H., Gorwood, P., Chignon, J.M., Adés, J., Lôo, H., 2000. [Factor analysis of french translation of the Barratt impulsivity scale (BIS-10)]. Can. J. Psychiatry Rev. Can. Psychiatr. 45, 156-165.

Beilock, S.L., Kulp, C.A., Holt, L.E., Carr, T.H., 2004. More on the fragility of performance: choking under pressure in mathematical problem solving. J. Exp. Psychol. Gen. 133, 584-600. doi:10.1037/0096-3445.133.4.584

Cardebat, D., Doyon, B., Puel, M., Goulet, P., Joanette, Y., 1990. [Formal and semantic lexical evocation in normal subjects. Performance and dynamics of production as a function of sex, age and educational level]. Acta Neurol. Belg. 90, 207-217.

Di Rosa, E., Schiff, S., Cagnolati, F., Mapelli, D., 2015. Motivation-cognition interaction: how feedback processing changes in healthy ageing and in Parkinson's disease. Aging Clin. Exp. Res. doi:10.1007/s40520-015-0358-8 


\section{ACCEPTED MANUSCRIPT}

Golden, C.J., 1978. Stroop color and word test, Chicago: Stoelting.

Hälbig, T.D., Tse, W., Frisina, P.G., Baker, B.R., Hollander, E., Shapiro, H., Tagliati, M., Koller, W.C., Olanow, C.W., 2009. Subthalamic deep brain stimulation and impulse control in Parkinson's disease. Eur. J. Neurol. 16, 493-497. doi:10.1111/j.1468-

1331.2008.02509.x

Hershey, T., Campbell, M.C., Videen, T.O., Lugar, H.M., Weaver, P.M., Hartlein, J., Karimi, M., Tabbal, S.D., Perlmutter, J.S., 2010. Mapping Go-No-Go performance within the subthalamic nucleus region. Brain 133, 3625-3634. doi:10.1093/brain/awq256

Hughes, A.J., Ben-Shlomo, Y., Daniel, S.E., Lees, A.J., 1992. What features improve the accuracy of clinical diagnosis in Parkinson's disease: a clinicopathologic study. Neurology 42, 1142-1146.

Jahanshahi, M., Obeso, I., Baunez, C., Alegre, M., Krack, P., 2015. Parkinson's disease, the subthalamic nucleus, inhibition, and impulsivity. Mov. Disord. Off. J. Mov. Disord. Soc. $30,128-140$. doi:10.1002/mds.26049

Kornblum, S., Hasbroucq, T., Osman, A., 1990. Dimensional overlap: cognitive basis for stimulus-response compatibility--a model and taxonomy. Psychol. Rev. 97, 253-270.

Marin, R.S., Biedrzycki, R.C., Firinciogullari, S., 1991. Reliability and validity of the Apathy Evaluation Scale. Psychiatry Res. 38, 143-162.

Mattis, S., 1988. Dementia rating scale professional manual, Odessa: Psychological Assessment Resources.

Mobbs, D., Hassabis, D., Seymour, B., Marchant, J.L., Weiskopf, N., Dolan, R.J., Frith, C.D., 2009. Choking on the money reward-based performance decrements are associated with midbrain activity. Psychol. Sci. 20, 955-962. doi:10.1111/j.1467-

9280.2009.02399.x 


\section{ACCEPTED MANUSCRIPT}

Montgomery, S.A., Asberg, M., 1979. A new depression scale designed to be sensitive to change. Br. J. Psychiatry J. Ment. Sci. 134, 382-389.

Nelson, H.E., 1976. A modified card sorting test sensitive to frontal lobe defects. Cortex 12, 313-324.

Padmala, S., Pessoa, L., 2011. Reward reduces conflict by enhancing attentional control and biasing visual cortical processing. J. Cogn. Neurosci. 23, 3419-3432. doi:10.1162/jocn_a_00011

Padmala, S., Pessoa, L., 2010. Interactions between cognition and motivation during response inhibition. Neuropsychologia 48, 558-565. doi:10.1016/j.neuropsychologia.2009.10.017

Palminteri, S., Serra, G., Buot, A., Schmidt, L., Welter, M.-L., Pessiglione, M., 2013. Hemispheric dissociation of reward processing in humans: insights from deep brain stimulation. Cortex J. Devoted Study Nerv. Syst. Behav. 49, 2834-2844. doi:10.1016/j.cortex.2013.02.014

Pinheiro, J., Bates, D., DebRoy, S., Sarkar, D., EISPACK authors, R-core, 2015. nlme: Linear and Nonlinear Mixed Effects Models.

Reitan, R.M., 1958. Validity of the trail making test as an indicator of organic brain damage. Percept. Mot. Skills 8, 271-276. doi:10.2466/pms.1958.8.3.271

Ridderinkhof, K.R., 2002a. Micro- and macro-adjustments of task set: activation and suppression in conflict tasks. Psychol. Res. 66, 312-323. doi:10.1007/s00426-0020104-7

Ridderinkhof, K.R., 2002b. Activation and suppression in conflict tasks: Empirical clarification through distributional analyses, in: Prinz, W., Hommel, B. (Eds.), Common 


\section{ACCEPTED MANUSCRIPT}

Mechanisms in Perception and Action (Attention and Performance Series). Oxford University Press, Oxford, pp. 494-519.

Ridderinkhof, K.R., Forstmann, B.U., Wylie, S.A., Burle, B., van den Wildenberg, W.P.M., 2011. Neurocognitive mechanisms of action control: resisting the call of the Sirens. Wiley Interdiscip. Rev. Cogn. Sci. 2, 174-192. doi:10.1002/wcs.99

Ridderinkhof, K.R., van den Wildenberg, W.P.M., Wijnen, J., Burle, B., 2004. Response inhibition in conflict tasks is revealed in delta plots, in: Posner, M.I. (Ed.), Cognitive Neuroscience of Attention, New York, Guilford Press, pp.369-377.

Schuepbach, W.M.M., Rau, J., Knudsen, K., Volkmann, J., Krack, P., Timmermann, L., Hälbig, T.D., Hesekamp, H., Navarro, S.M., Meier, N., Falk, D., Mehdorn, M., Paschen, S., Maarouf, M., Barbe, M.T., Fink, G.R., Kupsch, A., Gruber, D., Schneider, G.-H., Seigneuret, E., Kistner, A., Chaynes, P., Ory-Magne, F., Brefel Courbon, C., Vesper, J., Schnitzler, A., Wojtecki, L., Houeto, J.-L., Bataille, B., Maltête, D., Damier, P., Raoul, S., Sixel-Doering, F., Hellwig, D., Gharabaghi, A., Krüger, R., Pinsker, M.O., Amtage, F., Régis, J.-M., Witjas, T., Thobois, S., Mertens, P., Kloss, M., Hartmann, A., Oertel, W.H., Post, B., Speelman, H., Agid, Y., Schade-Brittinger, C., Deuschl, G., 2013.

Neurostimulation for Parkinson's Disease with Early Motor Complications. N. Engl. J. Med. 368, 610-622. doi:10.1056/NEJMoa1205158

Sheehan, D.V., Lecrubier, Y., Sheehan, K.H., Amorim, P., Janavs, J., Weiller, E., Hergueta, T., Baker, R., Dunbar, G.C., 1998. The Mini-International Neuropsychiatric Interview (M.I.N.I.): the development and validation of a structured diagnostic psychiatric interview for DSM-IV and ICD-10. J. Clin. Psychiatry 59 Suppl 20, 22-33-57. 


\section{ACCEPTED MANUSCRIPT}

Simon, J.R., 1990. The effects of an irrelevant directional cue on human information processing., in: Stimulus-response Compatibility: An Integrated Perspective, Amsterdam: North-Holland. R. W. Proctor \& T. G. Reeve, pp. 31-86.

Spielberger, C.D., 1983. State-trait anxiety inventory for adults, Palo Alto. ed, Consulting Psychologists Press.

van den Berg, B., Krebs, R.M., Lorist, M.M., Woldorff, M.G., 2014. Utilization of rewardprospect enhances preparatory attention and reduces stimulus conflict. Cogn. Affect. Behav. Neurosci. 14, 561-577. doi:10.3758/s13415-014-0281-z

van Wouwe, N.C., van den Wildenberg, W.P.M., Claassen, D.O., Kanoff, K., Bashore, T.R., Wylie, S.A., 2014. Speed pressure in conflict situations impedes inhibitory action control in Parkinson's disease. Biol. Psychol. 101, 44-60. doi:10.1016/j.biopsycho.2014.07.002

Wagenbreth, C., Zaehle, T., Galazky, I., Voges, J., Guitart-Masip, M., Heinze, H.-J., Düzel, E., 2015. Deep brain stimulation of the subthalamic nucleus modulates reward processing and action selection in Parkinson patients. J. Neurol. 262, 1541-1547. doi:10.1007/s00415-015-7749-9

Welter, M.L., Houeto, J.L., Tezenas du Montcel, S., Mesnage, V., Bonnet, A.M., Pillon, B., Arnulf, I., Pidoux, B., Dormont, D., Cornu, P., Agid, Y., 2002. Clinical predictive factors of subthalamic stimulation in Parkinson's disease. Brain J. Neurol. 125, 575-583. Wylie, S.A., Ridderinkhof, K.R., Bashore, T.R., van den Wildenberg, W.P.M., 2010a. The effect of Parkinson's disease on the dynamics of on-line and proactive cognitive control during action selection. J. Cogn. Neurosci. 22, 2058-2073.

doi:10.1162/jocn.2009.21326 


\section{ACCEPTED MANUSCRIPT}

Wylie, S.A., Ridderinkhof, K.R., Elias, W.J., Frysinger, R.C., Bashore, T.R., Downs, K.E., van Wouwe, N.C., van den Wildenberg, W.P.M., 2010b. Subthalamic nucleus stimulation influences expression and suppression of impulsive behaviour in Parkinson's disease. Brain J. Neurol. 133, 3611-3624. doi:10.1093/brain/awq239

Wylie, S.A., van den Wildenberg, W.P.M., Ridderinkhof, K.R., Bashore, T.R., Powell, V.D., Manning, C.A., Wooten, G.F., 2009. The effect of Parkinson's disease on interference control during action selection. Neuropsychologia 47, 145-157. doi:10.1016/j.neuropsychologia.2008.08.001

Zavala, B., Zaghloul, K., Brown, P., 2015. The subthalamic nucleus, oscillations, and conflict. Mov. Disord. 30, 328-338. doi:10.1002/mds.26072 


\section{ACCEPTED MANUSCRIPT}

highlights

Action control in incentive context with STN-DBS 